CERN-EP/99-98

July 131999

\title{
Charge Collection Efficiency and Resolution of an Irradiated Double Sided Silicon Microstrip Detector Operated at Cryogenic Temperatures
}

\author{
Author List \\ K. Borer, S. Janos, V.G. Palmieri ${ }^{1}$, \\ Laboratorium für Hocherergiephysik der Universität Bern, Sidlerstr.5, CH 3012 Bern, \\ Switzerland
}

J. Buytaert, V. Chabaud, P. Chochula, P. Collins ${ }^{2}$, H. Dijkstra² ${ }^{2}$ T.O. Niinikoski, C Lourenço,

C. Parkes, S. Saladino, T. Ruf

CERN, CH-1211, Geneva 23, Switzerland

V. Granata ${ }^{3}$, S. Pagano, F. Vitobello

Istituto di Cibernetica del CNR, Via Toiano 6, 80072, Arco Felice, Italy

W. Bell

Department of Physics and Astronomy, University of Glasgow, Glasgow G12 8QQ, UK

P. Bartalini, O. Dormond, R. Frei

Institut de Physique des Hautes Energies, Batiment des Sciences Physiques, Université de Lausanne, CH-1015 Dorigny 20, Switzerland

L. Casagrande

LIP, av. E. Garcia 14, P-1000, Lisbon, Portugal

T. Bowcock

Department of Physics, Oliver Lodge Laboratory, Liverpool, L69 7ZE, UK

I.B.M. Barnett

Institut für Kernphysik, Johannes Gutenberg-Universität Mainz, Johann-Joachim-Becher-Weg 45, D-55099 Germany

C. Da Via'4, I. Konorov, S. Paul, L. Schmitt

Physik Department E18, Technische Universität München, James Franck Strasse, D-85748 Garching, Germany

G. Ruggiero

Universita' Frederico II di Napoli, Dipartimento di Fisica and INFN, via Cinthia 1, Monte Sant'Angelo, Napoli, Italy

I. Stavitski

Dipartimento di Fisica, Università di Padova and INFN, Via Marzolo 8, I-35131 Padova, Italy

A. Esposito

Electrotechnical Laboratory,Electron Devices Division,1-1-4 Umezono, Tsukuba,305-8568 Ibaraki, JAPAN

\footnotetext{
${ }^{1}$ currently at CERN, CH-1211, Geneva 23, Switzerland

${ }^{2}$ Corresponding authors: Paula.Collins@cern.ch, Hans.Dijkstra@cern.ch

${ }^{3}$ also at Brunel University, Brunel, UK

${ }^{4}$ currently at Brunel University, Brunel, UK
} 


\begin{abstract}
This paper presents results on the measurement of the cluster shapes, resolution and charge collection efficiency of a double sided silicon microstrip detector after irradiation with $24 \mathrm{GeV}$ protons to a fluence of $3.5 \times 10^{14} \mathrm{p} / \mathrm{cm}^{2}$ and operated at cryogenic temperatures. An empirical model is presented which describes the expected cluster shapes as a function of depletion depth, and is shown to agree with the data. It is observed that the clusters on the $p$-side broaden if the detector is under-depleted, leading to a degradation of resolution and efficiency. The model is used to make predictions for detector types envisaged for the LHC experiments. The results also show that at cryogenic temperature the charge collection efficiency varies depending on the operating conditions of the detector and can reach values of $100 \%$ at unexpectedly low bias voltage. By analysing the cluster shapes it is shown that these variations are due to changes in depletion depth. This phenomenon, known as the "Lazarus effect", can be related to similar recent observations on diode behaviour.
\end{abstract}




\section{Contents}

1 Introduction 4

2 Description of the DELPHI Module 4

3 Irradiation of the Detector $\quad 5$

4 Operation in the Test-Beam 5

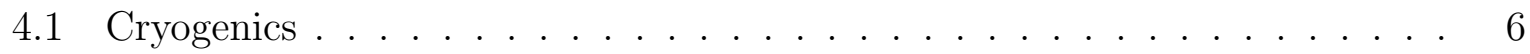

4.2 Data Acquisition . . . . . . . . . . . . . . . . . . . . 7

4.3 Data Summary . . . . . . . . . . . . . . . . . 7

5 Analysis Methods $\quad 7$

5.1 Charge Collection Measurement . . . . . . . . . . . . . . 7

5.2 Chip Calibrations . . . . . . . . . . . . . . . . . . . . . . . . . . . . . . . . . . 10

5.3 Alignment . . . . . . . . . . . . . . . . . . . . . 12

6 Cluster Shape Model $\quad 12$

7 Charge Collection Efficiency Results $\quad 15$

7.1 CCE and Depletion Depth . . . . . . . . . . . . . . 15

7.2 CCE Dependence on Operating Conditions . . . . . . . . . . . . . 17

7.2 .1 Time Dependence . . . . . . . . . . . . . . . . . . . . . . . . . . . . .

7.2.2 Starting Conditions Dependence . . . . . . . . . . . . . . 20

7.2.3 Temperature Dependence . . . . . . . . . . . . . . . . 20

7.2 .4 Bias Voltage Dependence . . . . . . . . . . . . . . . . 20

7.3 Discussion of CCE Results . . . . . . . . . . . . . . . . . . . . . 23

7.4 Reverse Bias Operation with Charge Injection . . . . . . . . . . . . . 24

8 Resolution Results $\quad 26$

8.1 Discussion . . . . . . . . . . . . . . . . . . . . 29

9 Conclusions $\quad 30$ 


\section{Introduction}

This paper reports on the performance of a double sided silicon strip detector after irradiation with $24 \mathrm{GeV}$ protons to fluence of $3.5 \times 10^{14} \mathrm{p} / \mathrm{cm}^{2}$. Following recent measurements carried out on heavily irradiated diodes [1] which indicated that a recovery of the charge collection efficiency (CCE) was possible at temperatures below $\sim 160 \mathrm{~K}$, the detector was operated at cryogenic temperatures. An extensive set of new measurements on diodes irradiated up to fluence equivalents of $2 \times 10^{15} 1 \mathrm{MeV} \mathrm{n} / \mathrm{cm}^{2}$ and operated at cryogenic temperatures may be found in the accompanying paper [2].

The use of double sided strip detectors extends the amount of information from that available from diodes alone, by allowing a measurement of the cluster shapes on both the ohmic $(n)$ and junction $(p)$ sides of the detector. Depending on the width of the depletion layer in the detector the cluster shapes change significantly, due to the changes in the electric field penetration. In this paper we describe a simple empirical model which describes the cluster shape as a function of depletion depth. As there is a well known relationship between incomplete depletion and drop in CCE, the measured cluster shape provides a powerful cross check on the measured CCE. The use of the cluster shape to distinguish between cases of complete and incomplete depletion is particularly useful for the analysis of heavily irradiated detectors, where the depletion voltage rises rapidly with the incident fluence and may not be well known for all situations. The model is used to analyse the CCE results obtained at cryogenic temperatures.

The changes in cluster shape have the consequence that for a realistic detector the resolution on the $p$-side will be degraded for situations of incomplete depletion. In this paper we address this point, comparing the measured resolutions with those predicted by the model.

This paper is organised as follows. Firstly the experimental setup is described. Then we discuss the methods by which the detector is aligned and the CCE is measured. In section 6 we present the model used to predict the cluster shapes. Section 7 presents the CCE and resolution results, and compares them with the predictions of the model. There is a presentation of results obtained in a partially forward biased situation, which show the detector operating in a completely different regime. In the final sections the implications of these results for the design of LHC silicon detectors are discussed.

\section{Description of the DELPHI Module}

The DELPHI module used was a rejected half module of the 1994 DELPHI [3] vertex detector, described in detail in [4]. The module is illustrated schematically in figure 1. It consists of two AC coupled double sided plaquettes with a sensitive area of $3.2 \times 5.4 \mathrm{~cm}^{2}$, daisy chained together and bonded to a double sided hybrid. The daisy chaining connects the capacitively coupled readout lines of the $p$-strips of one plaquette to read-out lines of the $n$-strips of the second plaquette, a technique which was employed in DELPHI to equalise the noise on each side of the module and to remove ambiguities in reconstructed clusters. All electronics is kept at ground potential, which limits the maximum depletion voltage to around $90 \mathrm{~V}$ to avoid breakdown of the coupling capacitors between the implants and read-out lines. The hybrid is equipped with ten radiation soft MX6 [5] CMOS chips, however given the $12 \mathrm{~cm}$ length of the the module it proved possible to irradiate the tip 
of the silicon while preserving the electronics.

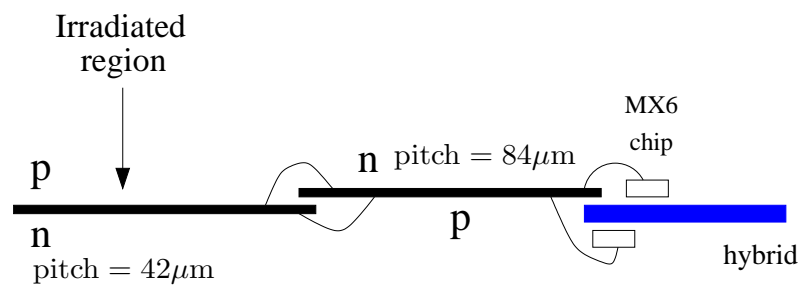

Figure 1: Schematic of the DELPHI module. Clusters from the two plaquettes can be distinguished by their sign.

The $p$-sides of the plaquettes have $12805.4 \mathrm{~cm}$ long strips with a pitch of $25 \mu \mathrm{m}$, with every second strip being read-out. The $n$-sides of the plaquettes have 1280 strips with a pitch of $42 \mu \mathrm{m}$, which run perpendicular to the $p$-side strips. The $n$-side signals are routed to the short side of the plaquette using a second metal layer. For the plaquette furthest away from to the hybrid the strip pitch is $42 \mu \mathrm{m}$ and each amplifier is connected to two strips, which leads to a twofold ambiguity in the position determination. For the plaquette closest to the hybrid neighbouring strips are connected together giving an effective $84 \mu \mathrm{m}$ strip pitch. The $n^{+}$strips are separated from each other with $p^{+}$blocking electrodes. The strips are biased via 10-20 $M \Omega$ polysilicon resistors. The plaquette furthest away from the hybrid has been irradiated. This plaquette was measured to be $310 \mu \mathrm{m}$ thick.

\section{Irradiation of the Detector}

The unbiased detector was irradiated at room temperature with $24 \mathrm{GeV}$ protons in the CERN-PS on August 13, 1998. By measuring the activation of a piece of aluminium, the average intensity in a $1 \mathrm{~cm}^{2}$ region was found to be $3.5 \times 10^{14}$ protons $/ \mathrm{cm}^{2}$. The irradiation was inhomogeneous, with the nominal maximum intensity centred on the detector at approximately $1 \mathrm{~cm}$ away from the tip. After irradiation the detector drew a total current, including the guard ring current, of $1 \mathrm{~mA}$ at $65 \mathrm{~V}$ at room temperature. The detector was stored at $-10^{\circ} \mathrm{C}$ until August 17th, 1998, and subsequently at a temperature between $25^{\circ} \mathrm{C}$ and $30^{\circ} \mathrm{C}$, until the test-beam, which started on August 26, when the detectors were cooled down to cryogenic temperatures.

After the test-beam the detector was stored at room temperature. Its current was remeasured 8 months later and found to be $20 \mu \mathrm{A}$ at $250 \mathrm{~K}$ with a bias voltage of $90 \mathrm{~V}$.

\section{Operation in the Test-Beam}

The test of the irradiated detector was performed in a $100 \mathrm{GeV} / \mathrm{c}$ muon test-beam of the CERN-SPS on August 29-30 1998. Tracks were reconstructed using a telescope consisting of 3 stations of silicon microstrip detectors, each measuring four coordinates $\left(x, y, 45^{\circ}\right.$ and $-45^{\circ}$ ). The muons were triggered with two scintillator planes upstream of the telescope. The telescope is fully described in [6]. The irradiated module was placed in a cryostat (described below) together with a non-irradiated reference module. This reference module 
is similar to the irradiated one, but consists of one plaquette only, placed back-to-back and downstream from the irradiated plaquette. The layout of the reference detector is the same as the plaquette close to the hybrid described in section 2 . The coordinate system is defined such that the $x$ coordinate is measured by the $p$-sides of the plaquettes and the $y$ coordinate by the $n$-sides of the plaquettes. The beam-test geometry is illustrated in figure 2. The tracks reconstructed by the telescope are extrapolated to the DELPHI plaquettes with an accuracy of approximately $17 \mu \mathrm{m}$ in the $x$ and $y$ coordinates.

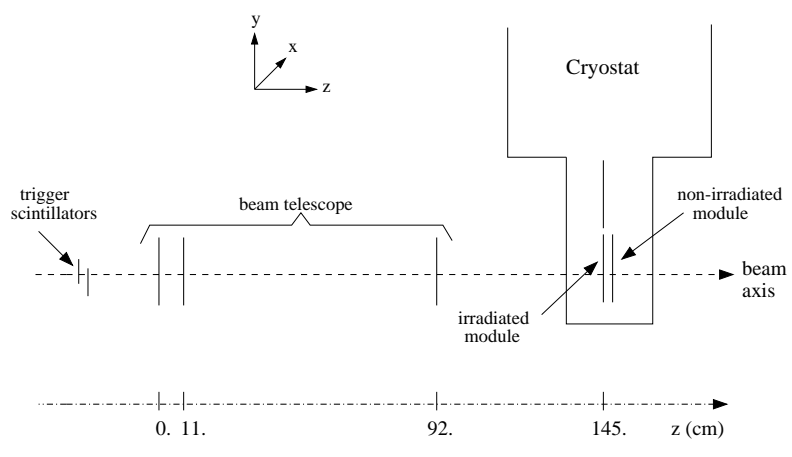

Figure 2: Schematic of the beamtest setup.

\subsection{Cryogenics}

The DELPHI modules were mounted on a top loading cryogenic insert, sealed in an Oxford Variox BL beam cryostat consisting of a liquid He reservoir with a shield cooled by liquid $\mathrm{N}_{2}$. The integrated variable temperature volume extends into a tail below the main body of the cryostat, equipped with thin aluminized mylar windows. Liquid He from the main reservoir passes through a needle valve and via a capillary tube to a heat exchanger surrounding the central sample tube. The temperature is controlled by the dual action of regulating both the flow of liquid He and the electrical power in a resistor in the heat exchanger.

The module was prepared for cryogenic operation by removing its ceramic side supports which otherwise would cause sufficient mechanical stress to break the detector. The DELPHI modules were not designed for cryogenic operation, and as a result the hybrid did not supply the operating voltages uniformly to the chips, which probably can be attributed to changing resistances at low temperature. This resulted in one non functioning chip on the reference detector, and one low gain chip on the irradiated detector. The 18 other readout chips functioned normally, as expected for CMOS circuits [7]. After the beam-test it was found that 50 bond wires out of a total of 1280 on the irradiated detector were detached.

At cryogenic temperatures the current in the detectors was $\lesssim 1 \mu \mathrm{A}$, giving a neglibible heat load in comparison to the MX6 chips. The temperature in the cryostat was continuously monitored with three PT100 resistors placed in the detector chamber and on each hybrid. After the beamtest another PT100 probe was glued onto the irradiated plaquette, and it was found that the temperature of the silicon was about 4 degrees below 
the hybrid temperature. All temperatures in this paper will refer to the temperature as measured on the hybrid of the irradiated detector.

\subsection{Data Acquisition}

The modules were controlled via a DELPHI repeater board placed just outside the cryostat, at a distance of about $1 \mathrm{~m}$. The integration time of the MX6 chips was set to $8 \mu \mathrm{s}$, and tracks were accepted if they traversed the detector within a $6 \mu$ s time interval, set $2 \mu \mathrm{s}$ before the end of the integration time. The data acquisition systems of the telescope and of the DELPHI detectors were independent and asynchronous, coupled by a common busy-logic. The data were merged off-line by using common event numbers and time-stamps scaled and recorded independently by each of the two systems.

\subsection{Data Summary}

The data presented in this paper were collected in a series of 32 runs, each lasting approximately 15 minutes. Table 1 shows the parameters of all the runs. In the first period, (runs 560 to 569), only the irradiated module was switched on and the voltage was scanned from $10 \mathrm{~V}$ to $90 \mathrm{~V}$ and back again in $20 \mathrm{~V}$ steps. In the second period (runs 570 to 582), the same scan was made but with the reference detector switched on to provide a precise point for resolution studies. The observed rise in temperature is the result of switching on the second module and of insufficient cooling power. In the final period, from run 588 onwards, both modules were switched on and the cooling power was increased. The current during runs 569, 570, 578 and 579 is due to partly forward biasing of the module, as explained in section 7.4.

All runs could be used to measure the charge collection efficiency, whereas the resolution studies used only data where both modules and the telescope were switched on. After the cuts described below, there were about 600 useful tracks traversing the modules per run. The beam defined by the scintillator telescope did not illuminate the whole silicon surface, but was distributed as shown in figure 3 .

\section{Analysis Methods}

\subsection{Charge Collection Measurement}

The charge deposited around a track was measured in one of three ways.

- Both modules operational (runs 570-578 and 581-597).

Events were selected if triggered in time with the telescope read-out system. In addition, each track was confirmed by associating with it a good $x$ or $y$ hit in the reference module. The track was then extrapolated to the irradiated module, and the signals on a chosen number of strips on either side of the extrapolated point were added to make the total signal. The signal pulse height distribution is smeared away from the ideal Landau distribution, firstly by the noise of the DELPHI module, which was approximately 1250 (1350) electrons per channel on the $p$-side ( $n$-side), and secondly by the fact that for certain runs the CCE changed during the run, as 


\begin{tabular}{|c|c|c|c|c|c|c|c|}
\hline $\begin{array}{l}\text { run } \\
\text { number }\end{array}$ & $\begin{array}{l}\mathrm{V}_{\text {bias }} \\
(\mathrm{V})\end{array}$ & $\begin{array}{c}\text { Current } \\
(\mu \mathrm{A})\end{array}$ & date & time & $\begin{array}{c}\text { temperature } \\
(\mathrm{K})\end{array}$ & Telescope & $\begin{array}{c}\text { Reference } \\
\text { Detector }\end{array}$ \\
\hline 560 & 10 & $<0.1$ & 29.09 .98 & 14h54:05-15h08:16 & 116.5 & $\mathrm{ON}$ & $\mathrm{OFF}$ \\
\hline 561 & 30 & $<0.1$ & 29.09 .98 & 15h10:26-15h24:49 & 117.5 & $\mathrm{ON}$ & OFF \\
\hline 562 & 50 & $<0.1$ & 29.09 .98 & 15h31:03-15h45:15 & 117.9 & $\mathrm{ON}$ & OFF \\
\hline 563 & 70 & 0.6 & 29.09 .98 & 15h52:24-16h06:51 & 118.4 & $\mathrm{ON}$ & OFF \\
\hline 564 & 90 & 1.3 & 29.09 .98 & 16h08:58-16h23:09 & 118.5 & $\mathrm{ON}$ & OFF \\
\hline 565 & 90 & 1.3 & 29.09 .98 & $16 \mathrm{~h} 29: 22-16 \mathrm{~h} 43: 33$ & 118.4 & $\mathrm{ON}$ & OFF \\
\hline 566 & 70 & 0.6 & 29.09 .98 & 16h49:46-17h03:56 & 118.5 & $\mathrm{ON}$ & OFF \\
\hline 567 & 50 & 0.1 & 29.09 .98 & 17h06:34-17h20:31 & 118.5 & $\mathrm{ON}$ & OFF \\
\hline 568 & 30 & $<0.1$ & 29.09 .98 & 17h23:54-17h38:03 & 118.5 & $\mathrm{ON}$ & OFF \\
\hline 569 & 13 & -1.3 & 29.09 .98 & 18h03:27-18h17:52 & 118.5 & $\mathrm{ON}$ & $\mathrm{OFF}$ \\
\hline 570 & 12 & -1.1 & 29.09 .98 & 18h46:10-19h00:05 & 119.4 & $\mathrm{ON}$ & $\mathrm{ON}$ \\
\hline 571 & 30 & $<0.1$ & 29.09 .98 & 19h08:58-19h22:53 & 122.8 & $\mathrm{ON}$ & $\mathrm{ON}$ \\
\hline 572 & 50 & 0.1 & 29.09 .98 & 19h33:26-19h47:36 & 124.1 & $\mathrm{ON}$ & $\mathrm{ON}$ \\
\hline 573 & 70 & 0.9 & 29.09 .98 & 19h50:16-20h05:22 & 125.8 & $\mathrm{ON}$ & $\mathrm{ON}$ \\
\hline 574 & 90 & 1.9 & 29.09 .98 & 20h11:07-20h25:17 & 126.3 & $\mathrm{ON}$ & $\mathrm{ON}$ \\
\hline 575 & 70 & 1.1 & 29.09 .98 & 20h27:41-20h42:34 & 127.5 & $\mathrm{ON}$ & $\mathrm{ON}$ \\
\hline 576 & 50 & 0.1 & 29.09 .98 & 20h45:41-21h00:48 & 128.3 & $\mathrm{ON}$ & $\mathrm{ON}$ \\
\hline 577 & 30 & $<0.1$ & 29.09 .98 & 21h02:58-21h17:07 & 128.8 & $\mathrm{ON}$ & $\mathrm{ON}$ \\
\hline 578 & 14 & -1.1 & 29.09 .98 & 21h20:44-21h35:07 & 129.3 & $\mathrm{ON}$ & $\mathrm{ON}$ \\
\hline 579 & 14 & -1.3 & 29.09 .98 & 21h55:03-22h08:58 & 144.2 & OFF & $\mathrm{ON}$ \\
\hline 581 & 50 & 0.1 & 29.09 .98 & $22 \mathrm{~h} 41: 36-22 \mathrm{~h} 55: 46$ & 149.5 & $\mathrm{ON}$ & $\mathrm{ON}$ \\
\hline 582 & 70 & 1.8 & 29.09 .98 & 23h00:48-23h10:24 & 149.8 & $\mathrm{ON}$ & $\mathrm{ON}$ \\
\hline 588 & 90 & 1.2 & 30.09 .98 & 4h22:39- 4h36:49 & 135.3 & $\mathrm{ON}$ & $\mathrm{ON}$ \\
\hline 589 & 90 & 1.2 & 30.09 .98 & 4h37:18- 4h51:13 & 135.9 & $\mathrm{ON}$ & $\mathrm{ON}$ \\
\hline 590 & 90 & 1.2 & 30.09 .98 & 4h51:42- 5h06:06 & 136.2 & $\mathrm{ON}$ & $\mathrm{ON}$ \\
\hline 591 & 90 & 1.2 & 30.09 .98 & 5h06:34- 5h20:44 & 136.2 & $\mathrm{ON}$ & $\mathrm{ON}$ \\
\hline 592 & 90 & 1.2 & 30.09 .98 & 5h21:13- 5h35:22 & 136.2 & $\mathrm{ON}$ & $\mathrm{ON}$ \\
\hline 593 & 90 & 1.2 & 30.09 .98 & 5h36:06- 5h50:01 & 136.2 & $\mathrm{ON}$ & $\mathrm{ON}$ \\
\hline 594 & 90 & 1.2 & 30.09 .98 & 5 h50:30- 6 h04:54 & 136.2 & $\mathrm{ON}$ & $\mathrm{ON}$ \\
\hline 595 & 90 & 1.2 & 30.09 .98 & 6h05:22- 6h19:32 & 136.2 & $\mathrm{ON}$ & $\mathrm{ON}$ \\
\hline 596 & 90 & 1.2 & 30.09 .98 & 6h20:01- 6h35:37 & 136.2 & $\mathrm{ON}$ & $\mathrm{ON}$ \\
\hline 597 & 90 & 1.2 & 30.09 .98 & 6h36:06- 6h50:01 & 136.3 & $\mathrm{ON}$ & $\mathrm{ON}$ \\
\hline
\end{tabular}

Table 1: Table of data collected during the test-beam run. 


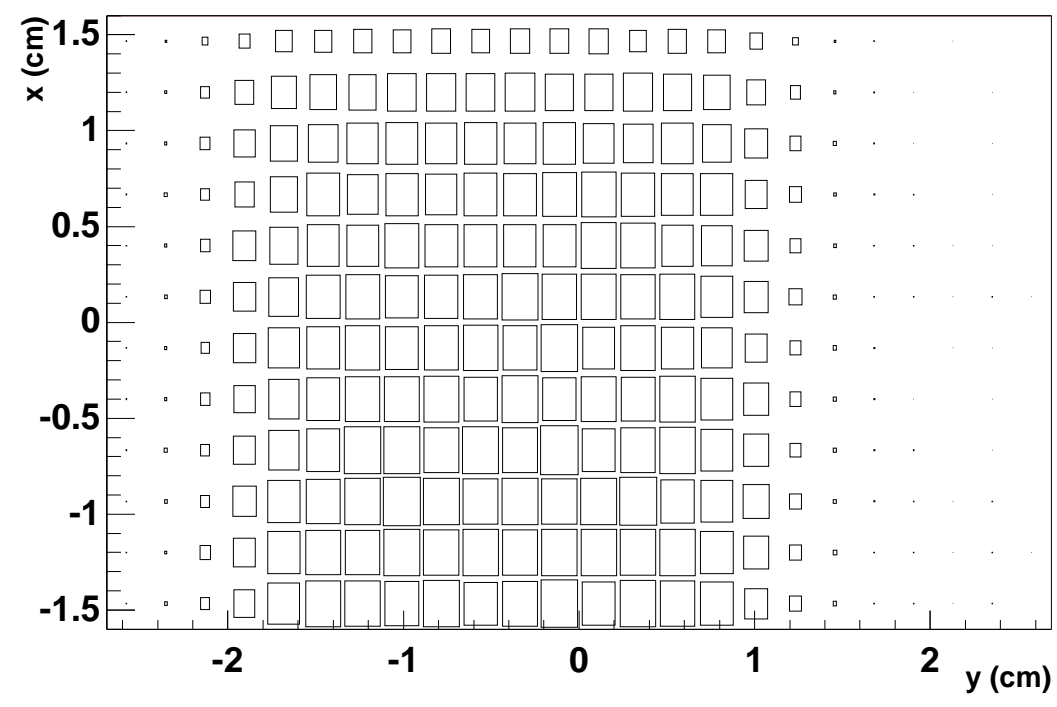

Figure 3: Illumination of the test-beam on the surface of the silicon plaquette. About 20,000 tracks traversed the plaquette in time with the DELPHI readout.

discussed in section 7.2.1. The pulse height distribution in ADC counts was fitted with the following function [8]:

$$
\text { pulseheight } \propto \exp -\left(\frac{(A D C-S)}{w}+\exp \left(\frac{S-A D C}{w}\right)\right)
$$

where $\mathrm{S}$ is the most probable energy loss of a minimum ionising particle (MIP), in ADC counts, and $w$ is allowed to be different on either side of the most probable energy loss. This function was found to be robust against the smearing effects. The shape of the fits is illustrated in figure 4 for signals integrated over four and eight strips.

- One module operational (runs 560-569).

When only the irradiated module was working the telescope tracks were simply extrapolated, and the events were required to be in time with the trigger. This was the case for the first 10 runs which were taken at the coldest temperatures. Studies with the runs where both modules were operational showed that this introduced a negligible impurity into the selection.

- No telescope information available (run 579).

In this run no track information was available from the telescope. A cluster search algorithm was applied to both modules. A good cluster was required to have at least one channel with a signal to noise ratio $(\mathrm{S} / \mathrm{N})$ of greater than four, with both neighbours having a smaller $\mathrm{S} / \mathrm{N}$. If exactly one cluster was found in both $x$ and $y$ in the reference module, an extrapolation was made to the likely impact point in the irradiated module with a resulting error on the position of the order of $16 \mu \mathrm{m}$ due 
to the beam divergence of $1.5 \mathrm{mrad}$, and to the $1.1 \mathrm{~cm}$ separation of the plaquettes. If a cluster candidate was found in the irradiated module within $100 \mu \mathrm{m}$ of this point in either coordinate its pulse-height was taken as the collected charge, and fitted with function 1 . Using this technique, the lower limit in $\mathrm{S} / \mathrm{N}$ which could be measured was around 5 .
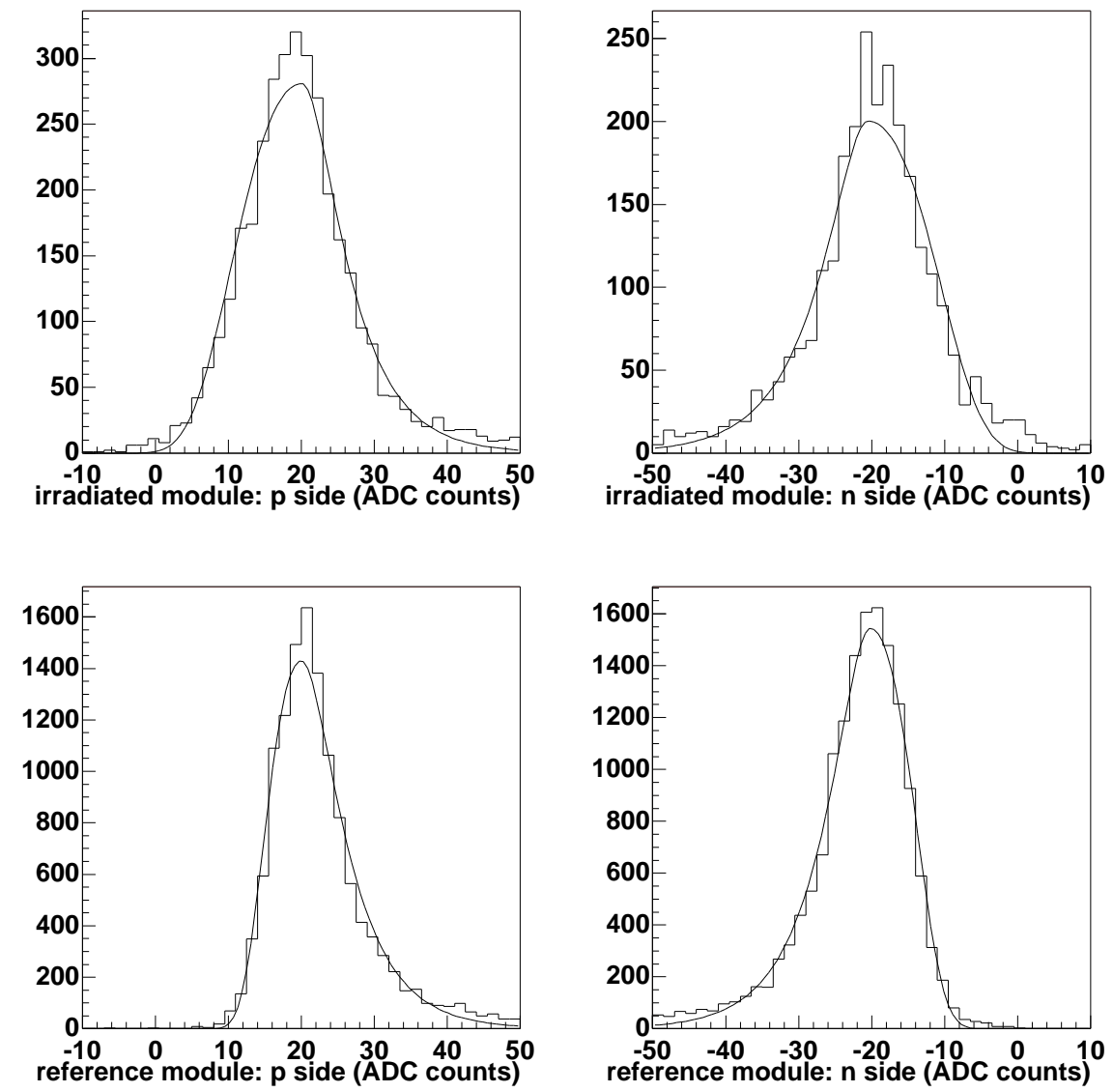

Figure 4: Example of pulse height distributions showing the fitted function described in the text. For the purposes of illustration a region of the plaquette is chosen which is far away from the nominal position of maximum irradiation intensity. For the irradiated module the charge is integrated over eight strips, and for the reference module the charge is integrated over four strips.

\subsection{Chip Calibrations}

Under the conditions of the test-beam, the voltage and current supplied to the chips differed from their values at room temperature. The voltage supplies were adjusted to achieve the best possible performance, but as the hybrid was not designed for operating at low temperature, it was not possible to have all chips working simultaneously. In addition 
the gain of the chips showed a greater variation than at room temperature. The gain of the chips was calibrated using tracks passing through the region which had suffered the least radiation, identified as described at the end of this section, for runs 590-597.

The most probable energy loss of a MIP as measured by each chip is shown in figure 5 . On both detectors the gain of the chips varied by around $10 \%$ for 9 chips. One chip on the reference detector was not functioning. One chip on the irradiated detector had an exceptionally low gain. This chip, which read out channels in a band of width $0.6 \mathrm{~cm}$ along the least irradiated edge of the plaquette, was excluded from the analysis. The similarity between the gains found for the irradiated and reference detector indicate that a possible bias from the gain correction in the irradiated detector due to any incomplete CCE in the selected region is less than $5 \%$.

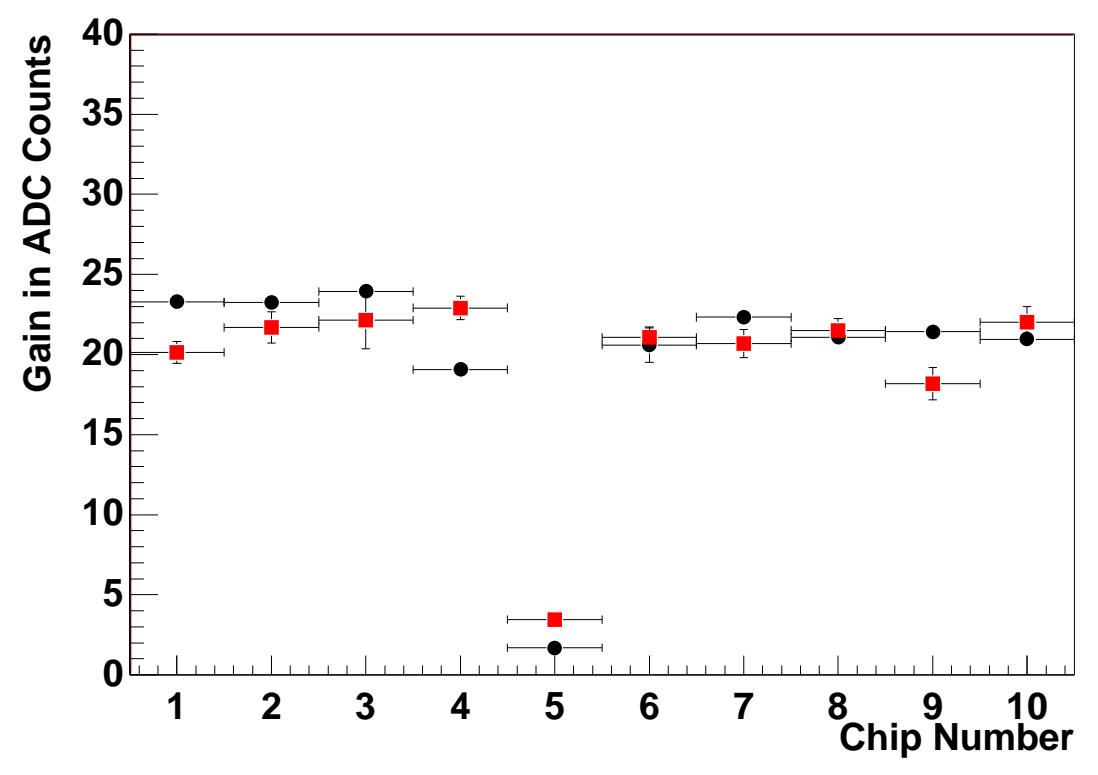

Figure 5: Fitted gain of the chips on the $p$-side (chips 1-5) and the $\mathrm{n}$ side (chips 6-10) for the reference (circles) and irradiated (squares) detectors.

The signals were then renormalised to these fitted gains in order to calculate the charge collection efficiencies. The eight-strip charge collection efficiency measured after these corrections in $2.5 \mathrm{~mm}^{2}$ sections of the irradiated plaquette is shown in figure 6 for runs 590-597. The reference plaquette is seen to be fully efficient in all regions, showing that the analysis method is reliable. The irradiated plaquette shows an area of reduced CCE, centred close to the nominal maximum position of the irradiation.

Using the data taken from the long stable running period of runs 590-597 we define the damaged areas on the detector with various degrees of irradiation by dividing the detector into a number of equally populated regions ordered in terms of charge loss. In the subsequent analysis, the CCE was measured for each area and for each run or fraction of run, and then related to other quantities for the same area, such as resolution or depletion depth. In general, the number of reference areas used was three, as illustrated in figure 7 . In the description which follows, the black area shown in this plot is referred to 

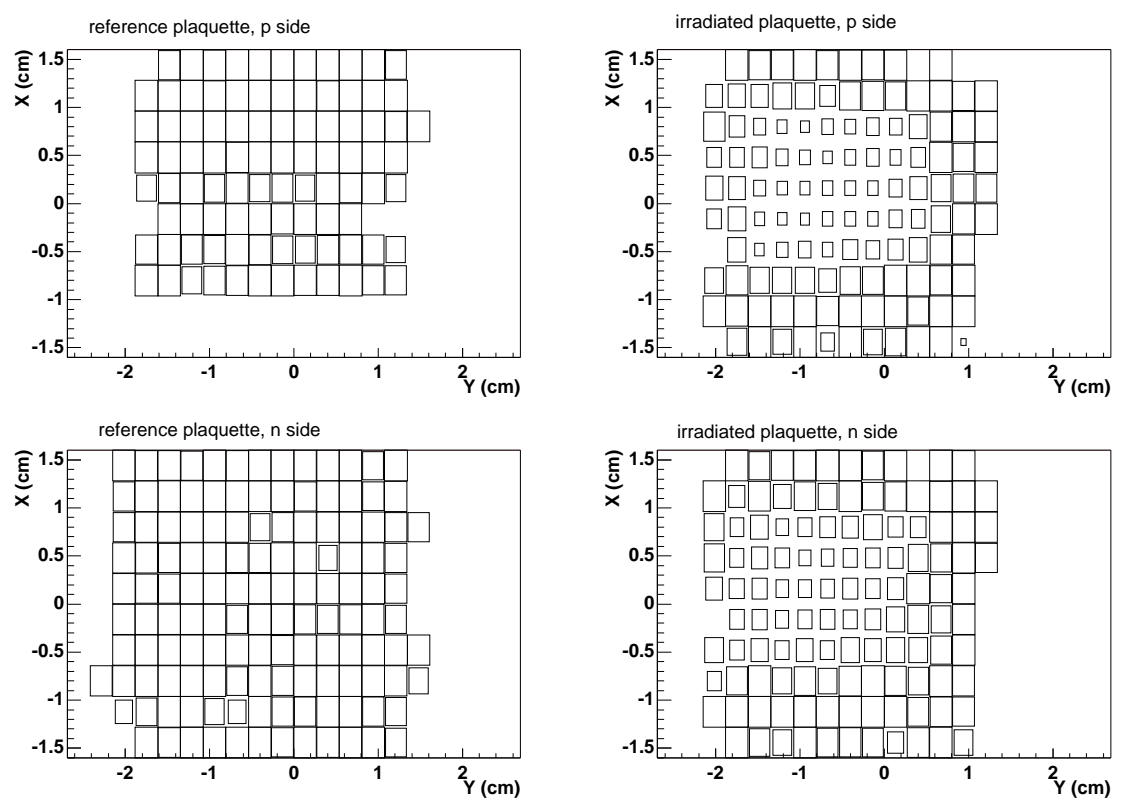

Figure 6: Fitted CCE for the runs 590-597 after the chip calibration procedure described in the text. A full square indicates 100\% CCE. The absence of a square indicates insufficient statistics to make a measurement. The empty band on the $p$-side of the reference plaquette indicates the region where there was a dead chip.

as the "hot-spot". For the long stable runs 590-597 finer divisions were used, for example 16 areas for the resolution studies.

\subsection{Alignment}

A variation in temperature of $30^{\circ} \mathrm{C}$ results in a vertical movement of the two DELPHI modules relative to the telescope of up to $200 \mu \mathrm{m}$, due to the top loading geometry of the cryostat. As a consequence, the irradiated module and the reference module should be aligned with respect to the telescope for each run or each series of consecutive runs taken at a constant temperature. Each module is given 7 degrees of freedom: 3 translations, 3 rotations and a thermal contraction factor. The alignment is performed minimising the sum of the $\chi 2$ of individual hits with respect to the corresponding tracks after discarding carefully the non gaussian tails.

The cluster position is reconstructed by taking the value of $\eta=L /(L+R)$, where $L$ and $R$ are the charge seen on the diode to the left and right of the track respectively.

\section{Cluster Shape Model}

In a heavily irradiated detector which has undergone type inversion, the depletion region grows with the applied voltage from the ohmic $(n)$ side of the detector. The non-depleted region reacts to an AC electrical signal like an insulator. The total charge collected after the passage of MIP has been summarised, for example, in [9]. The authors of this paper 


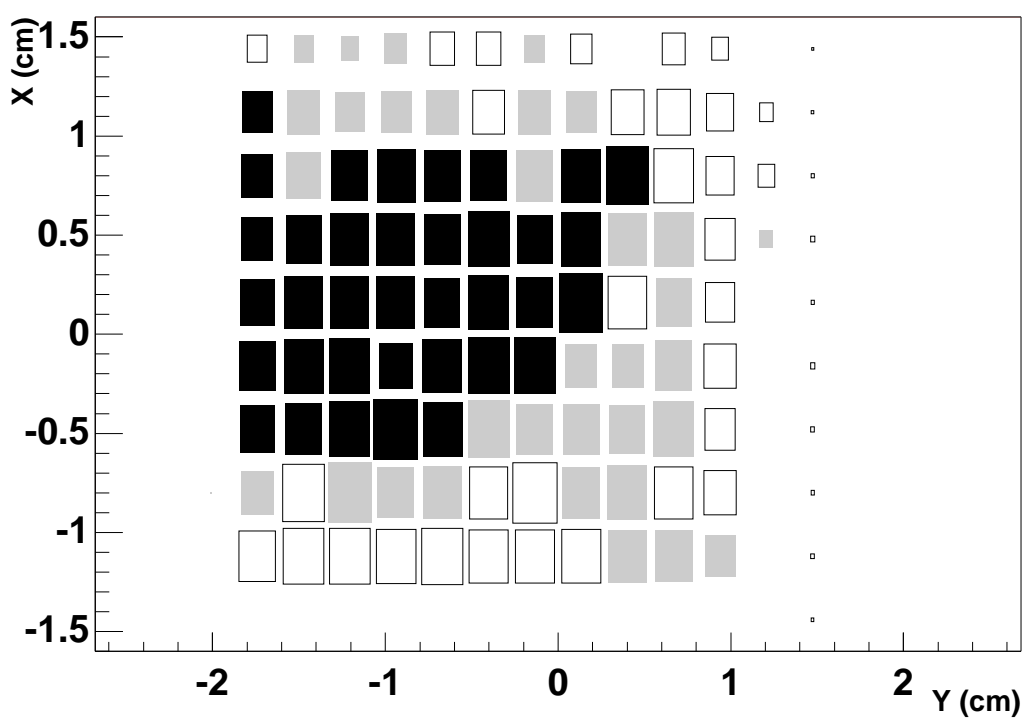

Figure 7: Division of the detector into 3 areas, using the information from runs 590-597. The black area corresponds to the most irradiated region, and is referred to as the "hotspot". The size of the squares shows the relative statistics available in each region after cuts. The empty areas were those excluded from the analysis due to noisy areas, or dead or low-gain channels. 
show that the CCE is proportional to $Q \times d / D$, where $Q$ is the total charge deposited in the active thickness $d$ of the detector, and $D$ is the total detector thickness. For a MIP, $Q$ is proportional to $d / D$, so the CCE is expected to scale with $d^{2} / D^{2}$, or with the bias voltage. It is possible to make a simple extension to this model to predict the spatial extent of the charge seen on the surface of the diode, and hence the resolution that can be expected for a strip detector. During the first few ns of the collection time, the electrons and holes released by the passage of the MIP drift along the electric field lines in a direction perpendicular to the detector plane. On the $n$-side the field lines bend towards the implants, and the track position is given by $\eta$. On the $p$-side the charges stop drifting when they reach the non-depleted region. During the first phase of the collection time we postulate that the charge seen on each diode is proportional to $1 / s^{2}$, where $s$ is the distance between the diode and the point at the edge of the non-depleted region where the track passed through. If the strip pitch is sufficiently fine with respect to the width of the non depleted region, this results in a significant spread of the resulting cluster. This is illustrated in figure 8 , where $s^{2}=x^{2}+(D-d)^{2}$. The left figure shows the case when the CCE is at $90 \%$, with a cluster shape close to normal. The right figure shows the contrasting situation when $25 \%$ of the detector thickness is non-depleted, corresponding to a CCE of $50 \%$, and hence to a much wider cluster. Clearly in order to compare the CCE with that from the diode measurements, it is necessary to integrate the signal on the $p$-side over a large number of strips.

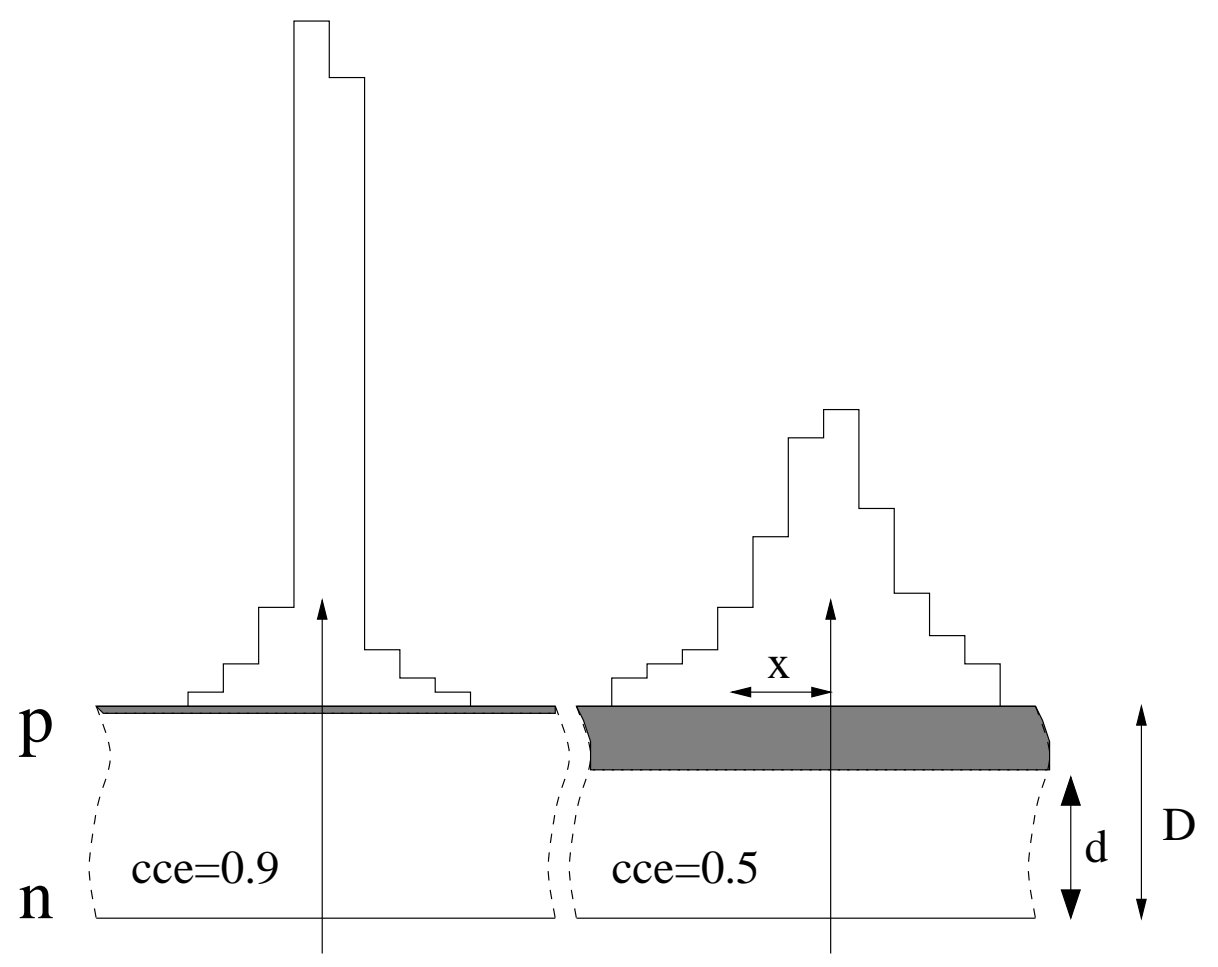

Figure 8: Sketch of the charge distribution on the $p$-side of the detector according to equation 2 for two depletion depths.

According to this empirical description, the fraction of charge collected by a strip which is separated by a distance $x$ from the traversing particle is equivalent to 


$$
f_{\mathrm{CCE}}=\frac{\frac{1}{x^{2}+(D-d)^{2}}}{\sum_{\text {strips }} \frac{1}{x^{2}+(D-d)^{2}}}
$$

The fit of this equation to the cluster shape gives directly $d$, the thickness of the depleted region. If all the charge loss can be attributed to incomplete depletion, then the equation $\mathrm{CCE}=d^{2} / D^{2}$ will hold. This is useful for the understanding of the CCE performance in irradiated detectors, where the voltage required to fully deplete the detector increases rapidly with the fluence. If there is a charge loss, it may be due to incomplete depletion, or to some other cause such as charge trapping. The measurement of the cluster shape distinguishes between these two cases.

Note that for realistic $\mathrm{S} / \mathrm{N}$ performance the cluster spread will seriously degrade the resolution. It is clear from figure 8 that applying the classic $\eta$ algorithm in the nondepleted case will always give a result for $\eta$ close to 0.5 . Figure 9 shows the $\eta$-distribution for the $p$-side clusters as a function of CCE as measured by integrating over 8 strips on the $n$-side for the runs 590-597. At low values of CCE the values of $\eta$ cluster around values of 0.5 due to the charge spreading. To get a more quantitative comparison between the model and the data the right hand plot shows the fraction of events with $|\eta-0.5|<0.2$ as a function of CCE. The full line is the result of a Monte-Carlo which smears the $p$-side clusters according to equation (2). For small CCE the noise starts to dominate and as a consequence the $\eta$-distribution will tend to become flat, which is the reason for the drop in the fraction of events with $\eta$ close to 0.5 for smaller values of CCE.

The Monte-Carlo assumes a Gaussian charge diffusion of $10 \mu \mathrm{m}$, it adds the appropriate electronic noise to the signals and it generates the total charge according to a Landau distribution. However, it does not attempt to model the electric field lines, nor Landau fluctuations along the track and it assumes all tracks to be perpendicular to the surface of the detector. As a result it finds a too optimistic resolution for fully depleted detectors.

The electrons and holes gradually recombine with free carriers arriving from the electrically neutral bulk. This process can lead to a further degradation of resolution for long integration times. With an integration time of $8 \mu$ s a charge loss due to slow collection times which might influence the CCE at 25 ns peaking time of the amplifier will not be observed in the data. In the empirical model developed in this paper these effects are ignored.

\section{Charge Collection Efficiency Results}

\subsection{CCE and Depletion Depth}

By plotting the CCE collected on two, four, six and eight strips around the impact point of the track, the cluster shape and overall CCE can be extracted. Looking at runs 590597 it is clear that the clusters have very different shapes in the different regions of the plaquette. Figure 10(a) shows a region where the CCE is more than $95 \%$ on the $n$-side of the plaquette. On the $p$-side of the plaquette the cluster is almost fully contained within four strips. When the charge loss is larger, as in plots 10(b), (c) and (d), the cluster becomes wider, and even integrating over 8 strips is not enough to recover the full CCE on the $p$-side. The solid lines show the fit to equation 2 , assuming that CCE $=d^{2} / D^{2}$. The data show a good agreement with the model. Note that the fit is a single parameter 

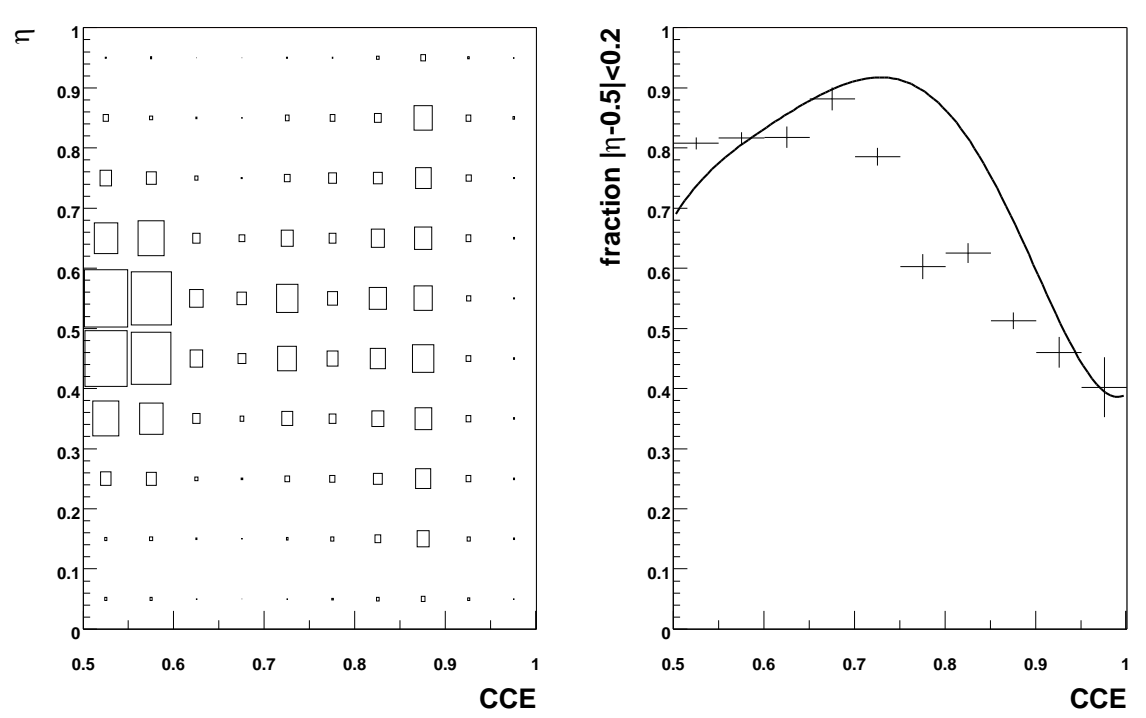

Figure 9: The $\eta$-distribution for the $p$-side clusters as a function of CCE measured on the $n$-side. The right plot shows the fraction of events with $|\eta-0.5|<0.2$ as a function of CCE. The full line shows the expectation as explained in the text.

fit which predicts not only the cluster shape but also the absolute level of the CCE, hence it is clear that the $n$-side data are incompatible with being on the non-depleted side. The dotted line shows the result of a two parameter fit, where the depletion depth and the absolute CCE level were allowed to vary independently. The result of the two fits are very similar. The worst agreement is seen for the most efficient area (smallest non-depleted region) where it may be expected that diffusion effects not accounted for in the model become more important.

From the fits the value of $d / D$, the fraction of the plaquette thickness which is depleted, can be extracted. In figure 11 (a) the CCE, as measured on the $n$-side of the plaquette integrating over 8 strips, is displayed as a function of the fitted value of $d^{2} / D^{2}$ as inferred from the $p$-side cluster shape, for six different regions on the plaquette, for runs 590-597. The correlation is very good, indicating that the measured values of CCE are indeed due to incomplete depletion and that the total CCE on $n$ and $p$-sides of the plaquette is equal. The fitted depletion depth is shown both for the one-parameter fit (full circles) and the two parameter fit (open circles). In runs 560-582 each run lasted for 15 minutes only, and it was found that the CCE changed during the run (see section 7.2.1). For this reason the measurements of CCE and depletion depth were made separately for each half of the run. Figure 11(b) shows, for these data, the CCE measured on the $n$-side as a function of the depletion depth in the "hot-spot", fitted separately for each half of runs 560-582 (excluding runs 569, 570, 578 and 579, which are discussed separately in section 7.4). The CCE of the "hot-spot" during runs 590-597, for comparison, was about $60 \%$. It can be seen that for this, the most irradiated area, the value of the CCE varied dramatically between the different runs, in some cases rising to values much higher than during the long stable period. This is discussed further in section 7.2. However at all times, the 

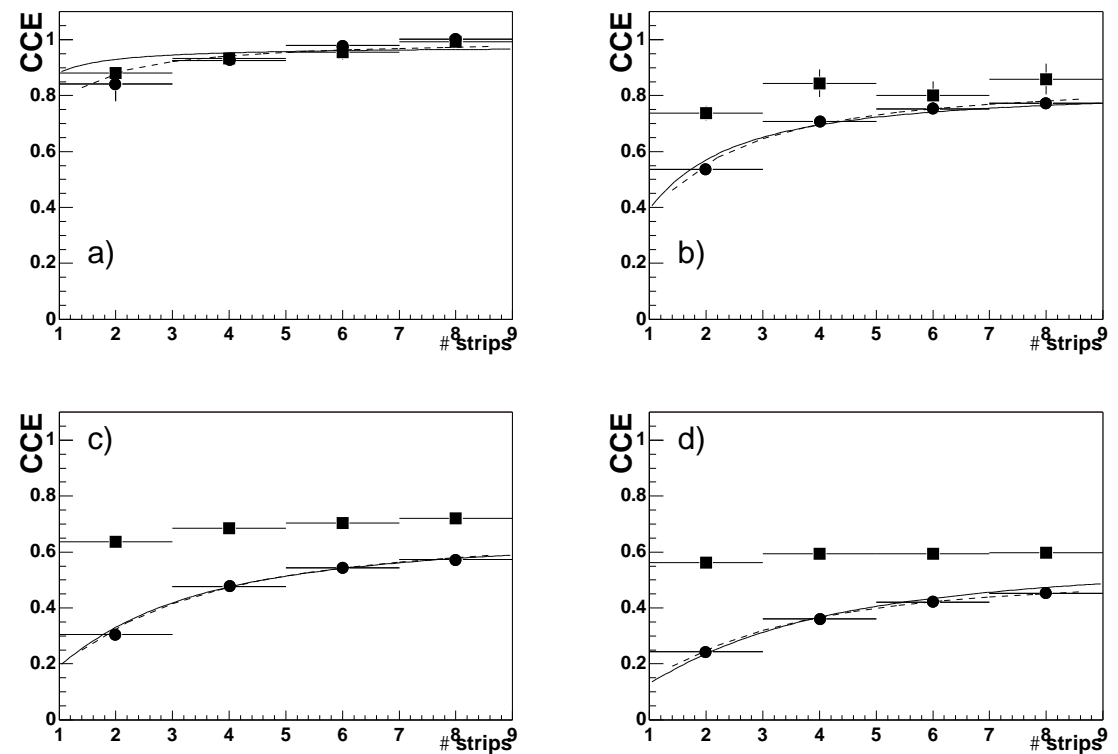

Figure 10: Cluster CCE as a function of number of strips included in the cluster, for $n$-side clusters (squares) and $p$-side clusters (circles) in four different regions of the irradiated plaquette. The solid lines show the result of the one parameter fit to equation 2 and the dashed lines show the result of the two parameter fit.

measured CCE is reflected in the fitted depletion depth. There is a greater spread in this plot than in figure 11(a), and this can be attributed to the fact that the measurements were made during less stable periods, and for a slightly larger area with consequently more spread in the CCE.

Following these observations, we define $\mathrm{CCE}$ as the charge integrated over eight strips on the $n$-side of the detector divided by the charge which would be expected for a MIP in a fully depleted detector. This wide cluster definition guarantees that the complete charge collection efficiency is measured, allowing comparisons with diode data. This will be referred to explicitly as $\operatorname{CCE}(n$-side).

\subsection{CCE Dependence on Operating Conditions}

In this section we discuss the measured values of CCE in the "hot-spot" in more detail. It was found to depend on four factors: time from the start of run, initial starting conditions, temperature and bias voltage.

\subsubsection{Time Dependence}

As was shown in section 7.1 there are some periods of running where the "hot-spot" showed a marked improvement in CCE. An example of this occurs in runs 564-565, which were two consecutive runs taken over a period of 35 minutes, with a bias voltage of $90 \mathrm{~V}$ and at a temperature of $118 \mathrm{~K}$. Figure 12 shows the corresponding $\mathrm{CCE}(n$-side) in the "hot-spot" as a function of time, together with the CCE expected from the depletion 


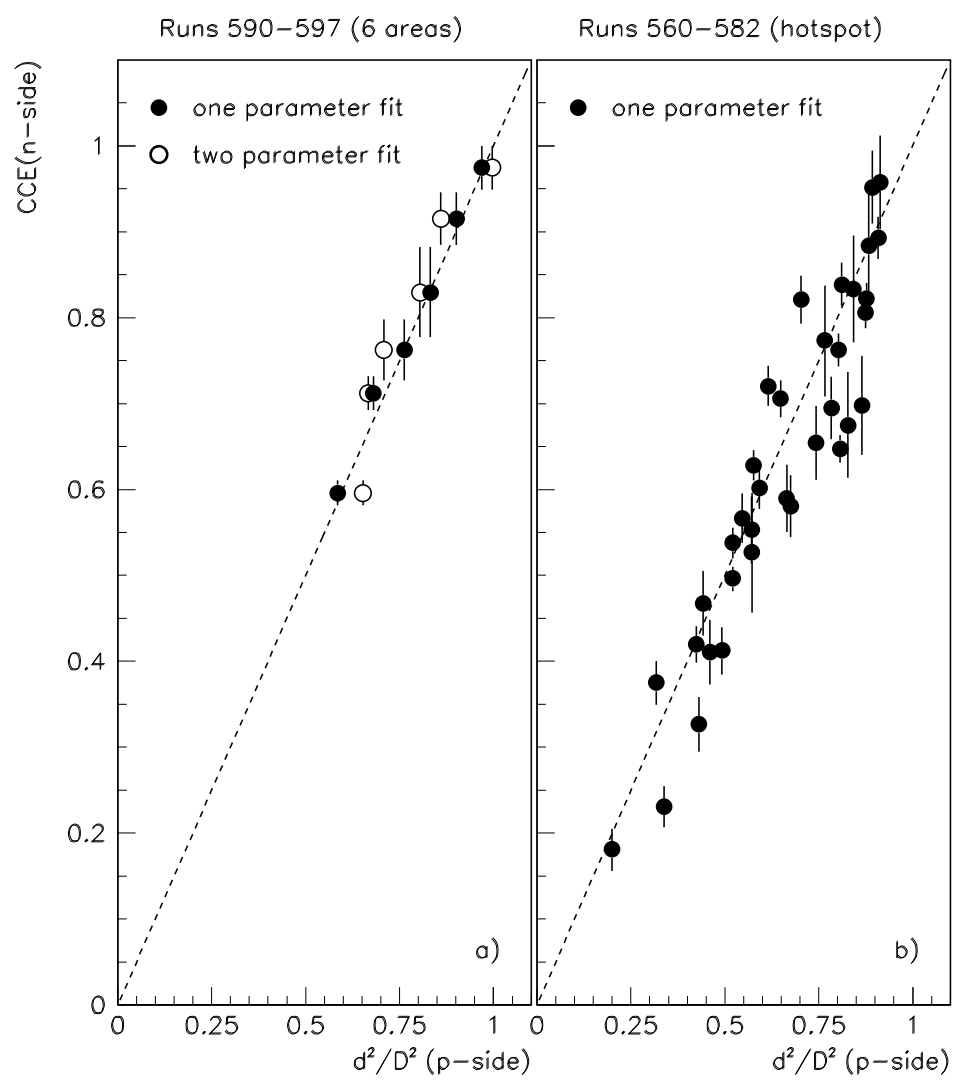

Figure 11: CCE measured on the $n$-side of the detector integrated over 8 strips plotted against $d^{2} / D^{2}$ measured from the cluster shape on the $p$-side of the detector. (a) is for runs 590-597, with a separate fit made for each of six regions of the detector. The solid circles show the result of the one-parameter fit and the open circles the result of the two-parameter fit. The dashed line shows the expected distribution if the CCE loss is dominated by incomplete depletion. (b) shows the result of the one-parameter fit to the "hot-spot" for runs 560-582 (excluding the forward-biased runs). The fit is made separately for the first and second half of each run due to the fact that the CCE changed rapidly during the run. 
depth as measured from the cluster shape on the $p$-side. For the first few minutes of the run a remarkable recovery is seen in both the CCE and the depletion depth. It appears that the voltage which was not sufficient to deplete the detector at higher temperatures is adequate to fully deplete the volume at a slightly lower temperature. With time this effect becomes less marked, and after 40 minutes the depletion depth has shrunk from more than $95 \%$ to $\sim 85 \%$ of the volume. From this data we cannot comment on the subsequent time evolution.

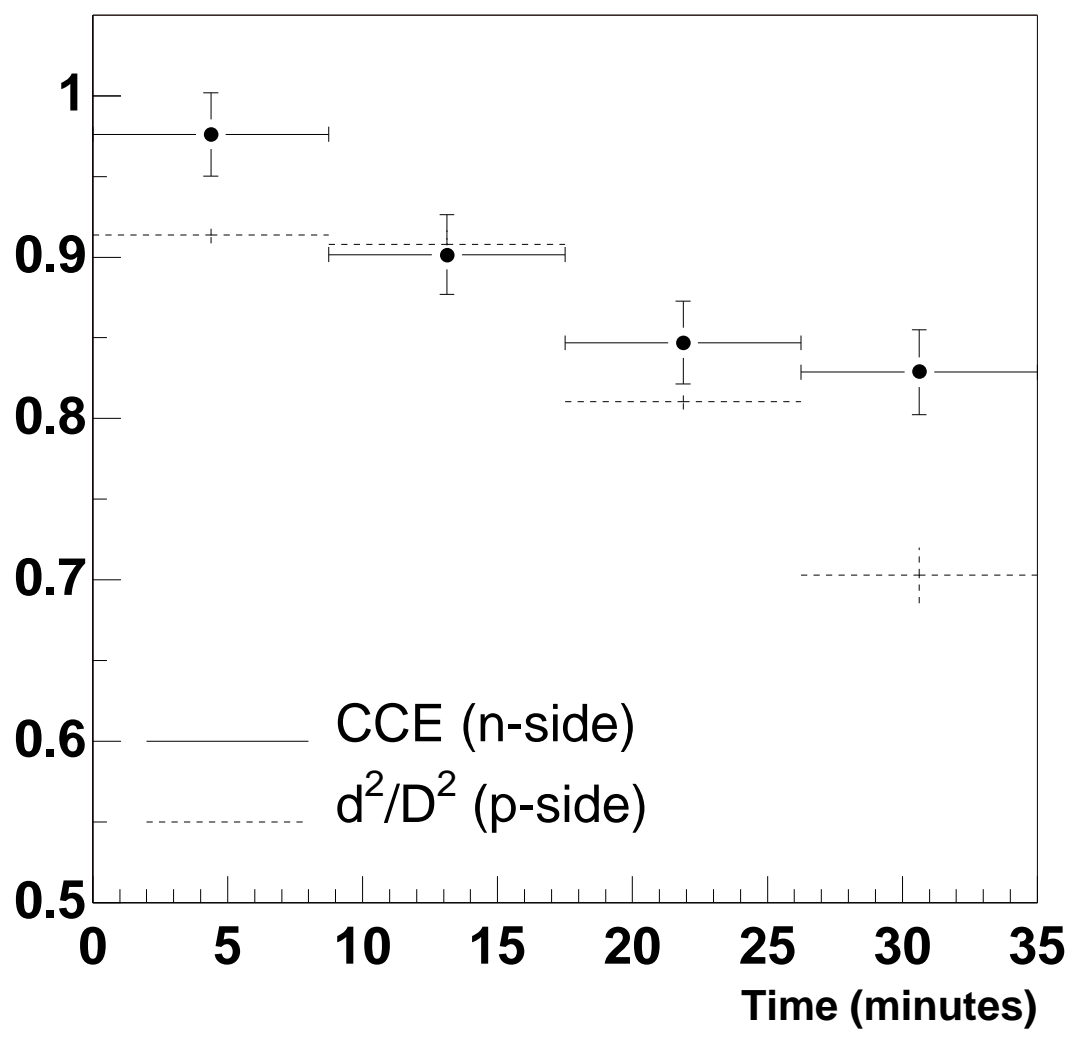

Figure 12: $\operatorname{CCE}\left(n\right.$-side) (circles) and $d^{2} / D^{2}$ (dashed line) of the "hot-spot", where $d$ is the depletion depth as measured on the $p$-side of the detector, as a function of time, for runs 564 and 565 .

A time dependence can also be seen for runs 588-597, which were continuous runs lasting a total of 150 minutes. Figure 13 shows for these data the $\mathrm{CCE}(n$-side) in the "hot-spot" as a function of time. The same plot also shows $d^{2} / D^{2}$ as measured from the $p$-side clusters. There is a good agreement between the curves, indicating that the CCE loss is due to the incomplete depletion. We also note that over the time of the run, there is a steady decrease in the thickness of the depleted region. Note that the decrease is fastest in the first 30 minutes, i.e. runs 588 and 589, which for this reason were not used in the definition of the areas described in section 5.2. 


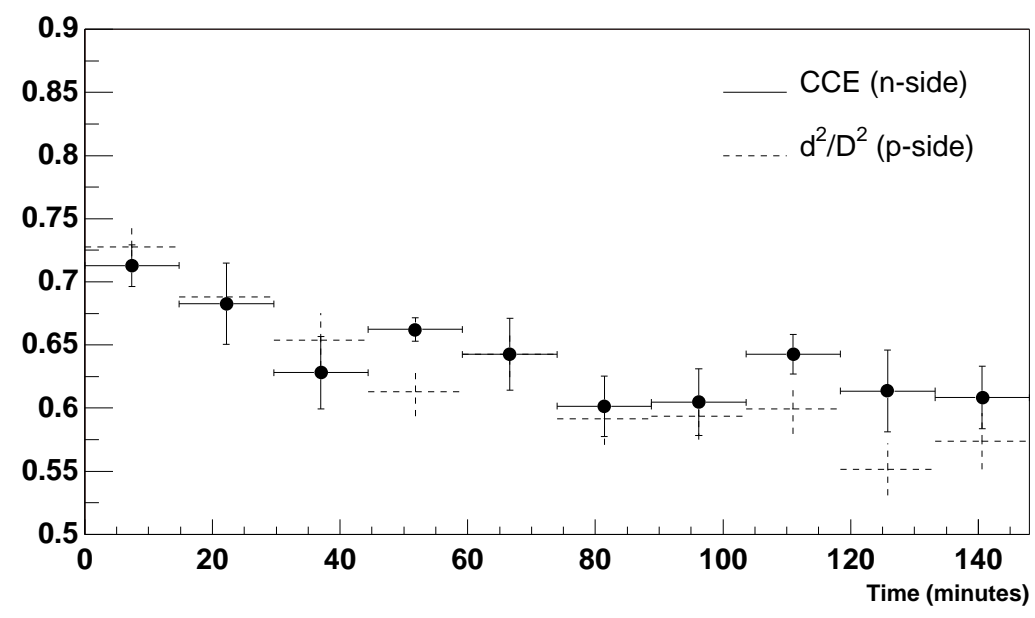

Figure 13: $\operatorname{CCE}\left(n\right.$-side) (circles) and $d^{2} / D^{2}$ (dashed line) of the "hot-spot", where $d$ is the depletion depth as measured on the $p$-side of the detector, as a function of time, for runs 588-597.

\subsubsection{Starting Conditions Dependence}

In figure 14 we show the $\operatorname{CCE}(n$-side) of the "hot-spot" as a function of time for all the short runs at temperatures below 120K, which lasted about 15 minutes each. It is clear from these plots that the value of the CCE was not exactly reproducible from one run at a certain bias voltage to the next. However one feature is evident. If the bias voltage was increased (decreased) before the start of run, indicated by the full (dashed) lines in the figure, then the CCE decreases (increases) as a function of time, and starts from a higher (lower) value. The range of CCE which is measured at a bias voltage of $30 \mathrm{~V}$ is particularly striking. These results show that at low temperatures, not only the time, but also the history of bias voltage applied to the detector influences the CCE.

\subsubsection{Temperature Dependence}

In the previous section we have seen that the time and the starting conditions affect significantly the CCE in the "hot-spot". In order to extract the temperature dependence, we select data from the start of a run, for runs for which with the bias voltage is increased just before they are started. Figure 15 shows the $\operatorname{CCE}(n$-side $)$ as a function of temperature, for three different bias voltages. There is a clear temperature dependence, with the recovery in $\mathrm{CCE}$ seen at the lower temperatures.

\subsubsection{Bias Voltage Dependence}

The dependence on bias voltage is extracted by examining data from the runs with a temperature of less than 119K, only using the data collected during the first 5 minutes of each run. The result is shown in figure 16. The solid circles show the result when the bias voltage is increased just before the start of run. For $30 \mathrm{~V}$ and higher the CCE is around $90 \%$, and the voltage dependence is quite weak. The open circles show the case when 


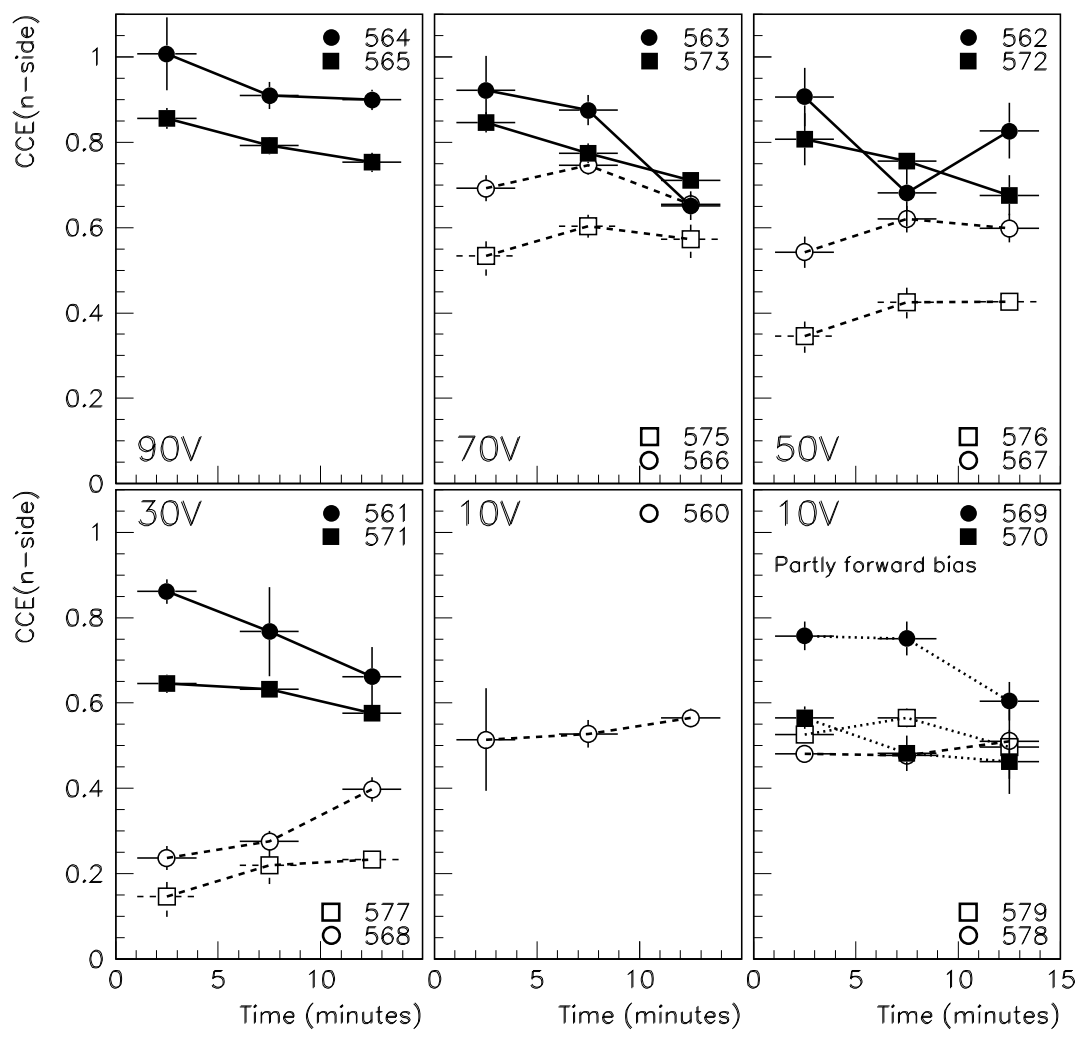

Figure 14: $\operatorname{CCE}(n$-side) of the "hot-spot" as a function of time for all the short runs. Lines on the histograms connect points from the same run. The solid (dashed) lines are for runs where the bias voltage was increased (decreased) immediately before the start of the run. For runs with a dotted line the history immediately before the start of run is not known. 


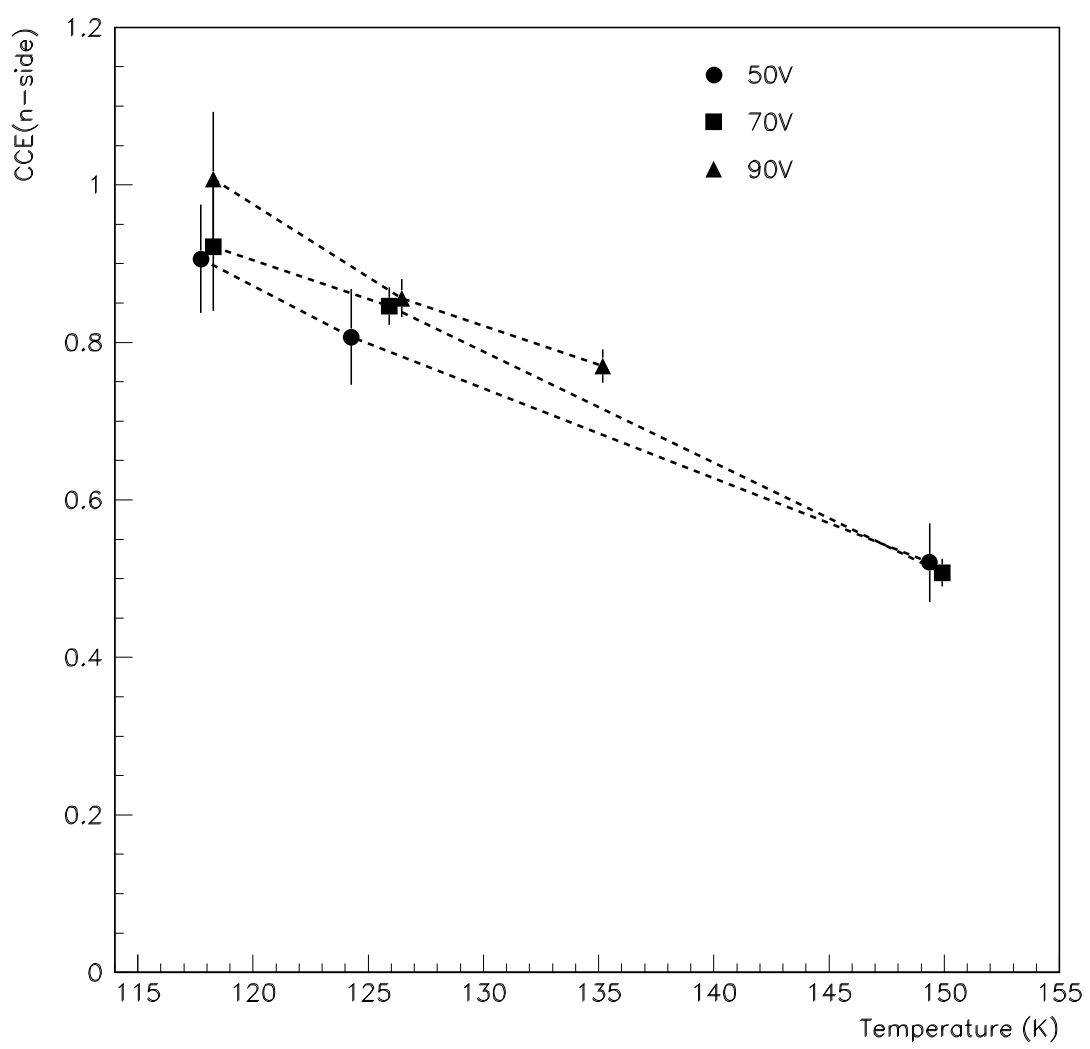

Figure 15: CCE(n-side) of the "hot-spot" measured during the first 5 minutes of runs as a function of temperature, for three bias voltages. 
the bias voltage is decreased just before the start of run, and here a stronger bias voltage dependence is observed.

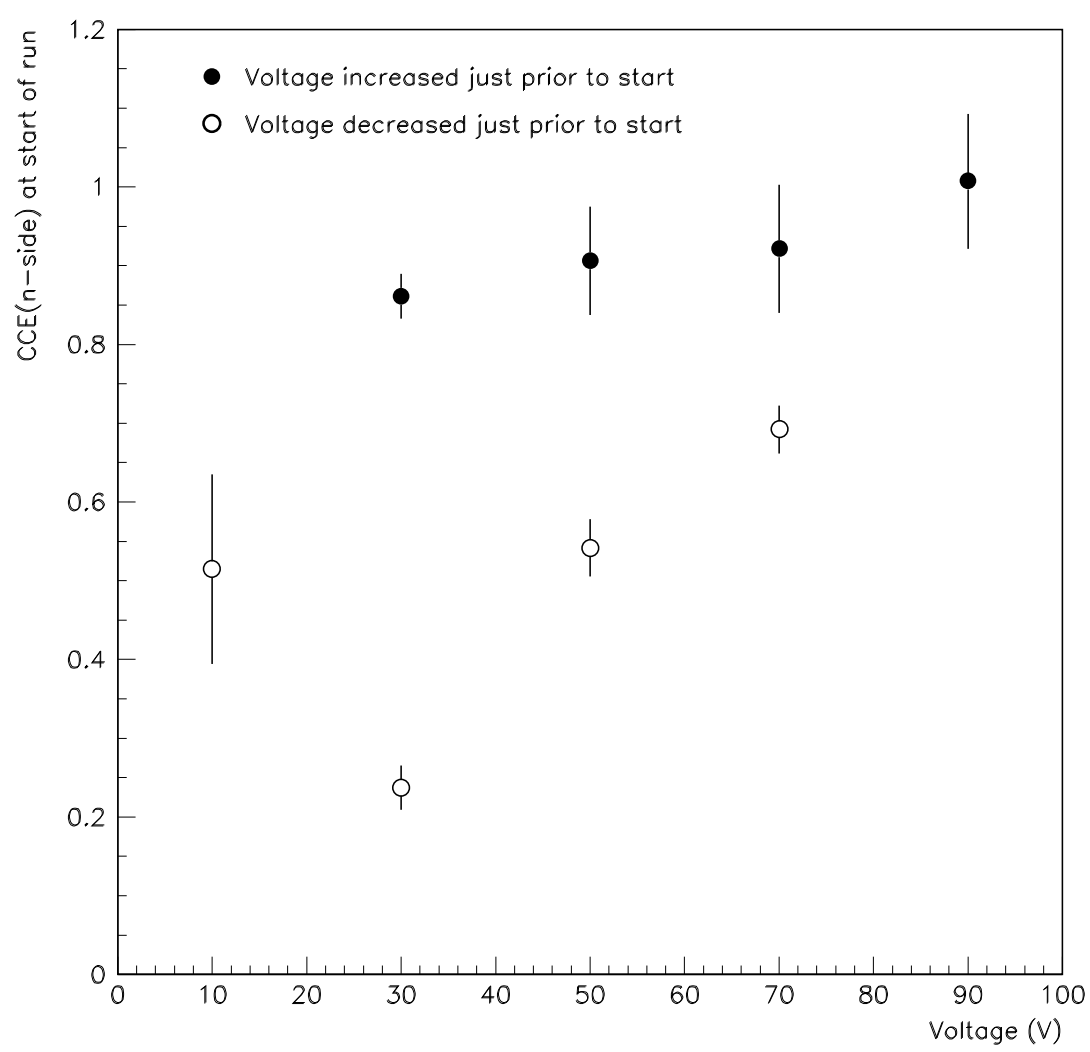

Figure 16: $\operatorname{CCE}(n$-side) of the "hot-spot" measured during the first 5 minutes of runs as a function of bias voltage, for runs below 119K.

\subsection{Discussion of CCE Results}

The results presented above can be summarised in terms of three points:

- The CCE of the most damaged area of the detector varies as a function of many parameters, chiefly temperature, time, bias voltage and bias voltage history.

- The CCE measured on the $n$-side has been shown to be always compatible with the depletion depth as measured from the cluster shapes on the $p$-side (see figure 11).

- A maximum CCE in the most irradiated region of $100 \%$ was observed temporarily (see figure 12).

These results can be understood in terms of a mechanism whereby the voltage necessary to deplete the detector depends on experimental conditions. Such a mechanism is the 
so called "Lazarus effect", which has been qualitatively discussed in [10]. The depletion voltage of a silicon detector is given by:

$$
V=\frac{q}{2 \epsilon_{o} \epsilon_{s}} \times D^{2} \times\left|N_{\mathrm{eff}}\right|
$$

where $q$ is the electron charge, $\epsilon_{s}$ the permittivity of silicon, $\epsilon_{o}$ the permittivity of free space, $D$ the detector thickness and $N_{\text {eff }}$ the effective doping density. For a detector which has undergone type inversion $N_{\text {eff }}$ is negative. For a fluence of $3.5 \times 10^{14} \mathrm{p} / \mathrm{cm}^{2}$ of 24 $\mathrm{GeV}$ the full depletion voltage is expected to be between about $150 \mathrm{~V}$ and $350 \mathrm{~V}[11,12]$, where the uncertainty comes from the lack of knowledge of the production details of this detector, and the difficulty of extrapolating diode measurements to strip detectors. The measurements on runs 590-598, displayed in figure 11(a) show that at $90 \mathrm{~V}$ the most irradiated region of the detector is indeed under-depleted. From the measured CCE of around $60 \%$ we deduce a full depletion voltage at $136 \mathrm{~K}$ of $150 \mathrm{~V}$.

The value of $N_{\text {eff }}$ can become less negative if hole traps are filled, for instance as a result of a current pulse in the silicon. This will result in a lowering of the full depletion voltage. At room temperature the trapping and de-trapping times are of the order of nanoseconds, whereas at cryogenic temperatures the de-trapping times become longer by many orders of magnitude [14]. The authors of [10] show that these long de-trapping times, or partial trap "freezing", make it possible to manipulate the value of $N_{\text {eff }}$. This was done in their case by injecting a hole current into bulk silicon to fill the hole traps and compensate the de-trapping. They show that there is a very delicate balance between the choice of current and temperature. Over-injection of charge can even lead to $N_{\text {eff }}$ becoming positive, and "re-inversion" of the detector.

For the data described here, the value of $N_{\text {eff }}$ was changing in an uncontrolled manner. When the bias voltage is increased just before the start of run, this leads to a recovery of the CCE. This may be attributed to a current pulse induced in the detector allowing hole traps to fill, and changing the value of $N_{\text {eff }}$ such that $90 \mathrm{~V}$ or even less is enough to deplete the detector. The measurement of the cluster shapes confirm that the CCE recovery can be understood in terms of changes in the depletion depth. Even at $30 \mathrm{~V}$ one can obtain almost full depletion, as measured at the start of run 561 (see figure 14). However this is clearly a dynamic situation, and the CCE recovery is lost after some minutes. Hence the "Lazarus effect" was observed, but it was not controlled. Further discussion on the control of the depletion voltage and CCE via injection of charge may be found in [2]. We note in addition that for the higher temperatures no CCE recovery was observed.

\subsection{Reverse Bias Operation with Charge Injection}

There is a set of runs, not discussed in the above, which are an exception to the characteristics described so far. For the runs 569, 570, 578 and 579, at the lowest bias voltage of around $10 \mathrm{~V}$ a significant forward current developed, as measured in the HV bias line of the irradiated plaquette. Note that for run 560, also a run at $10 \mathrm{~V}$, no such current was observed.

After the beamtest this forward current was reproduced and investigated in the laboratory. The DELPHI module contained 16 channels, randomly distributed over the detector, with a $\mathrm{DC}$ connection between the read-out line and the implant on the $n$-side of the plaquette closest to the hybrid. These read-out strips had been disconnected from 
the amplifier prior to the test-beam, and hence are at the potential of the bias voltage of this plaquette, which was kept at $60 \mathrm{~V}$ throughout the test-beam. Due to the fact that, as explained in section 2, the plaquettes are daisy chained together, these strips are connected to the $p$-side read-out strips of the plaquette furthest away from the hybrid, which is the irradiated plaquette under study. Sometime during the beamtest, in between runs 560 and 569, a DC connection between the $p$-implants and their read-out lines developed for these 16 channels on the irradiated plaquette. Hence, these $p$-implants, instead of being close to ground potential, have a potential somewhat lower than $60 \mathrm{~V}$, depending on the current per channel and its resistor network. For bias voltages on the irradiated plaquette of below $20 \mathrm{~V}$ these 16 channels cause locally a forward bias over about $3 \%$ of the total area. The effect is illustrated diagrammatically in figure 17 .

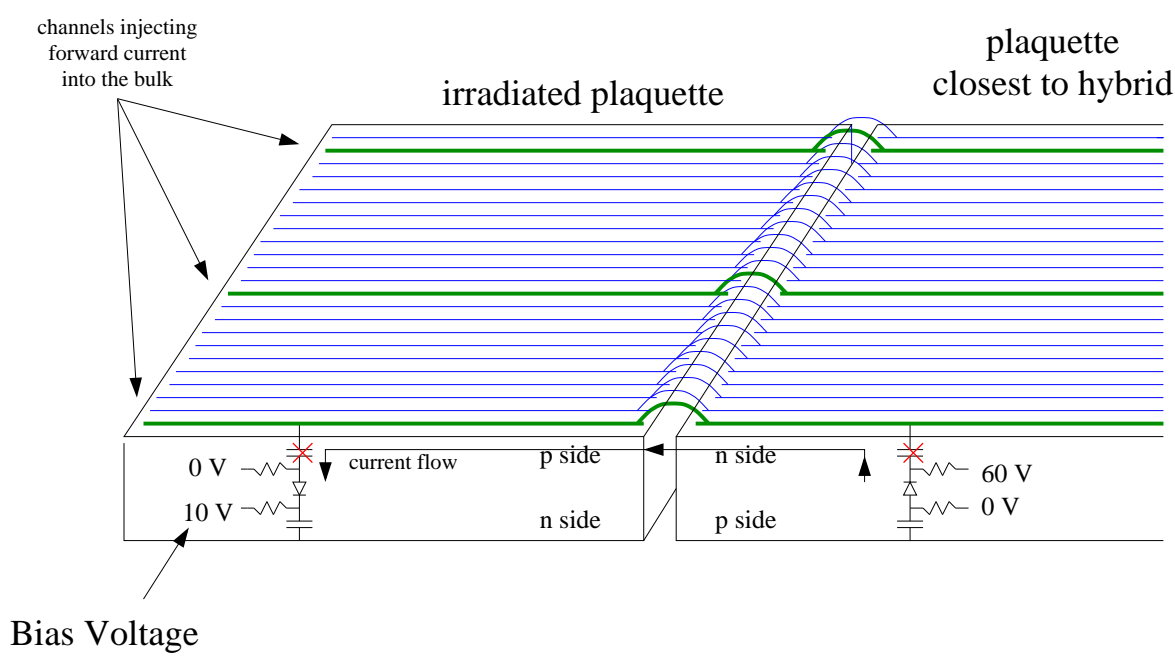

Figure 17: Diagram of the charge injection mechanism.

The CCE measured in the "hot-spot" for these four runs show no significant dependence on temperature or time (see figure 14). Figure 18(a) shows their CCE(n-side) as a function of $d^{2} / D^{2}$ in the "hot-spot", where $d$ is inferred from the $p$-side cluster shape fitting procedure explained above. For comparison the CCE of run 560 is also displayed. For each run $d^{2} / D^{2}$ is determined both with a one-parameter fit, and by allowing $d^{2} / D^{2}$ to vary independently from CCE (two-parameter fit). The fits give a similar result, showing that on the $p$-side the CCE agrees with the cluster shape. However the points show that the $\mathrm{CCE}(n$-side) has a significantly lower value than expected. Note that for the $10 \mathrm{~V}$ reverse biased run 560 this is not the case.

The discrepancy is clearly illustrated in figure 18(b), which shows the Landau distributions for the $n$ and $p$-side clusters integrating over eight channels for run 570 . A comparison of the cluster shapes on the $n$ and $p$-sides for this run is displayed in figure 18 (c), which shows that the charge on the $n$-side is confined to less than 4 channels, while on the $p$-side of the detector the spread is much larger, as is expected for incomplete depletion. All fitted $p$-side values for the reversed biased runs are above $75 \%$, which is a large CCE for such a low voltage, and also for temperatures above 140K. A possible explanation for the large CCE could be that by accident the $\sim 1 \mu \mathrm{A}$ injected by the forward bias over a small area of the detector caused a space charge compensation which lowered 
the necessary depletion voltage as explained in the previous section. However, we have no explanation for the discrepancy between the $n$ and $p$-side CCE, which is inconsistent with Ramo's theorem [16].
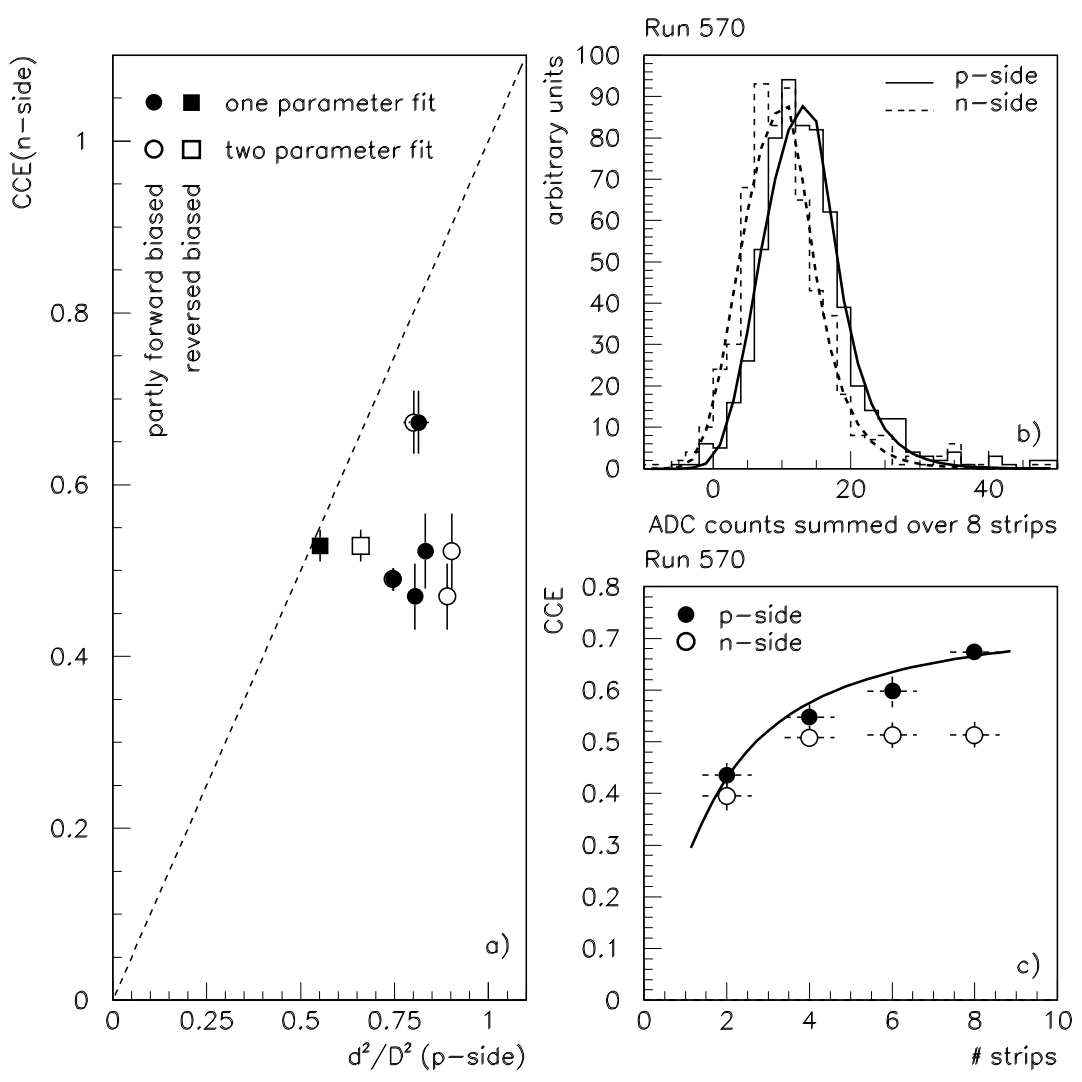

Figure 18: (a) $\operatorname{CCE}\left(n\right.$-side) for runs at $10 \mathrm{~V}$ as a function of $d^{2} / D^{2}$ as determined from the $p$-side cluster shape. The square marker is the data from run 560 , which was fully reversed biased, while the other markers are from runs with the detector being partly forward biased. (b) Landau distributions for eight channel wide clusters of run 570 with their fitted function according to equation (1). (c) Cluster shape for run 570. The smooth line is the result of a two parameter fit to equation (2).

\section{Resolution Results}

The beamtest setup provided three independent measurements of the position of a track per projection, thus allowing an unambiguous de-convolution of the resolutions from the individual detectors. The residual distributions between tracks and detector clusters were fitted with a Gaussian distribution. Influences from non-Gaussian tails were suppressed by including only the central $95 \%$ of the distributions in the fit. For the resolution study we have selected that part of the data which shows the smallest time dependence of the CCE. 
Runs 590-597 show only a small time dependence and were taken at a stable temperature. The large statistics allows the detector to be subdivided in sixteen areas with similar CCE per area as described in section 5.2. Figure 19 shows the resolution as measured on the $p$ and $n$-side of the DELPHI detectors as a function of the $\operatorname{CCE}(n$-side). The resolution of the tracks at the position of the DELPHI detectors as measured by the telescope was found to be $16.9 \pm 0.4 \mu \mathrm{m}$ in both coordinates. The resolution on the $p$-side of the reference detector was found to be around $5 \mu \mathrm{m}$, in agreement with the expectation for $25 \mu \mathrm{m}$ pitch with every second strip read out. The resolution on the $n$-side of the reference detector was found to be $19 \mu \mathrm{m}$, as expected for $84 \mu \mathrm{m}$ strip pitch and perpendicular tracks. The resolution on the $n$-side of the irradiated detector shows no dependence on the CCE. Since the S/N for full depletion was measured to be around 18, and the detector depletes from the $n$-side this is as expected. The measured resolution is around $12 \mu \mathrm{m}$, where one would expect around $9 \mu \mathrm{m}$ for a detector with $42 \mu \mathrm{m}$ strip pitch. The resolution on the $p$-side of the irradiated detector worsens rapidly with a decrease of CCE. Since the $\mathrm{S} / \mathrm{N}$ for the fully depleted detector is even slightly better than its $n$-side counterpart, this can not be attributed to a worse $\mathrm{S} / \mathrm{N}$. The dashed-line in Figure 19 is a prediction of the Monte Carlo described in section 6. It is clear that the model does describe this sharp increase, even showing quantitative agreement with the data. As was discussed before, for almost fully depleted regions the model does not attempt to simulate the correct field line map and charge division, hence predicting a too good resolution. The dotted curves show the resolution if one smears the prediction of the model with another $6 \mu \mathrm{m}$ to account for this defect.

An attempt was made to improve the resolution on the $p$-side by not using the $\eta$ algorithm to find the cluster position, but rather determining the cluster position by using the predicted shape of the cluster over three or five strips around the channel with the maximum signal. Since for a given CCE value this shape is known from the model, the position of the cluster is the only free parameter. Using this method on the Monte Carlo data yielded a marginal 10-15\% improvement of the resolution depending on the CCE.

A search was made for systematic shifts of the cluster position possibly caused by the non uniform irradiation over the detector surface. For each $5 \times 5 \mathrm{~mm}^{2}$ area on the irradiated detector the offset of the residual distributions was determined. No offset showed a significant deviation from zero, within the typical $2 \mu \mathrm{m}$ precision.

The resolution for the runs at lower bias voltages was measured taking into account only the second half of the run, where the CCE dependence on time and starting conditions is less marked. Due to limited statistics per run, we chose to subdivide the area of the detector in three regions with similar CCE values. Then the resolution is determined in the "hot-spot" area for every run, while using the resolutions of the reference detector and the telescope as determined with runs 590-597, as explained above. Figure 20 shows these resolutions for all the runs with both detectors and the telescope being operational. The resolution on the $n$-side is fully consistent with what was observed for the $90 \mathrm{~V}$ runs, i.e. no CCE dependence even for CCE values as low as $25 \%$. Hence $\mathrm{S} / \mathrm{N}$ is not an issue at these resolutions. On the $p$-side the resolution shows the variation with $\mathrm{CCE}$ as predicted by the model, represented with dashed and dotted lines, with the same convention as figure 19 .

The resolutions on the $p$-side of the $10 \mathrm{~V}$ runs, indicated with full circles, with $\mathrm{CCE}(n$ side) values around $50 \%$ depart from the general trend. However, as was shown in the 


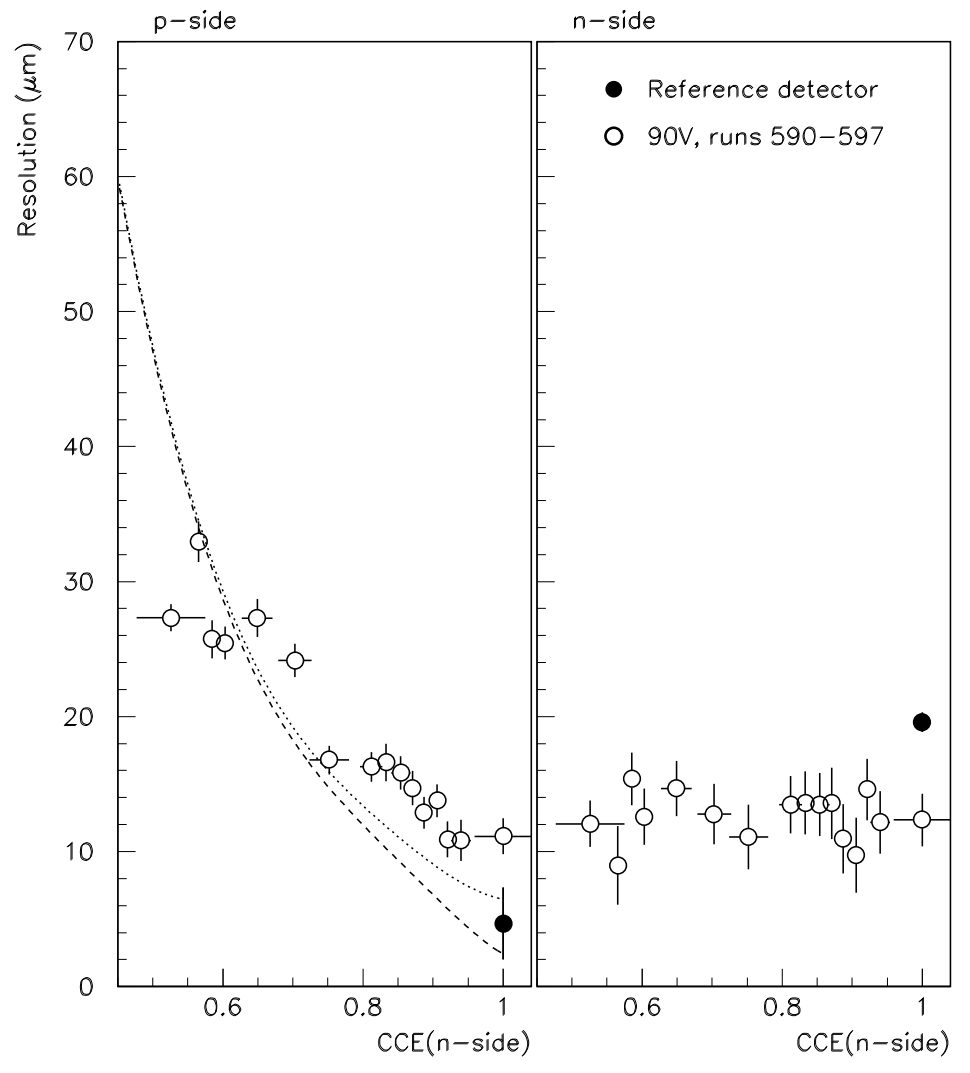

Figure 19: Resolution of runs 590-597 for the $p$-side and the $n$-side, as a function of $\operatorname{CCE}(n$-side). Note that the reference detector has an $n$-side pitch double that of the irradiated detector. 
previous section, for these runs the $n$-side CCE measurement is not in agreement with the $p$-side CCE as derived from the cluster shape. If we assume the CCE derived from the $p$-side cluster shape (75\%) rather than that from the $n$-side CCE (50\%), we find that our model is compatible with these resolutions.

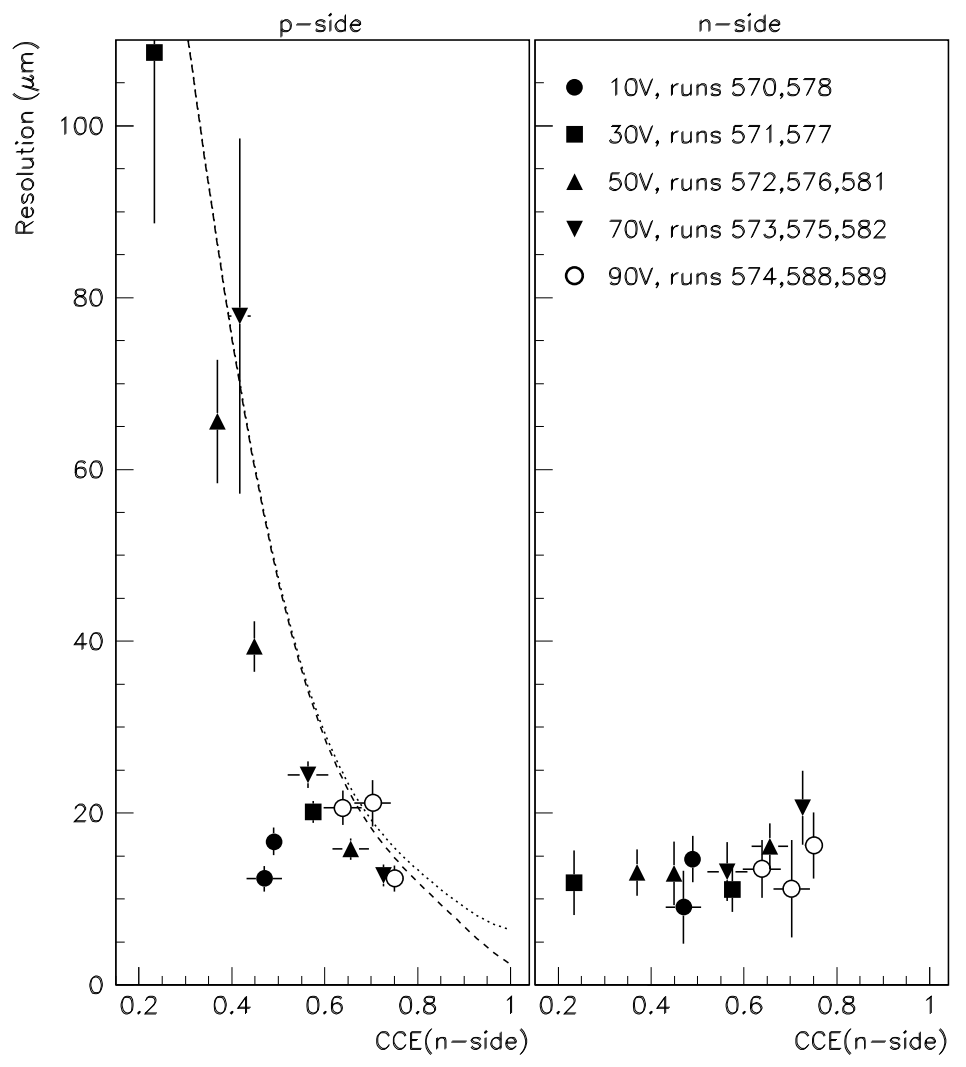

Figure 20: Resolution in the "hot-spot" of the second half of various runs for the $p$-side and the $n$-side, as a function of $\operatorname{CCE}(n$-side $)$.

\subsection{Discussion}

Having a model of the charge distribution allows a risk assessment in the choice of the detector types in the radiation environment of LHC, assuming that CCE losses are dominated by incomplete depletion.

Figure 21 shows an example of the application of the model for two cases. In figure 21(a) we show the result of modelling a detector which is $300 \mu \mathrm{m}$ thick, with an $80 \mu \mathrm{m}$ strip pitch and a binary read-out with a threshold of $6000 \mathrm{ENC}$ and a noise per channel of 1500 ENC, i.e. an ATLAS type detector. It can be clearly seen that in the case of a $p$-on- $n$ detector, the efficiency for finding a cluster above threshold degrades quickly if only $80 \%$ of the depletion voltage can be achieved, while for a $n$-on- $n$ design even at $50 \%$ of the depletion voltage the efficiency is quite acceptable. Furthermore it is interesting to 
note that with the $p$-on- $n$ design a slight improvement is expected in the resolution for perpendicular tracks when running about $10 \%$ under-depleted due to the smearing of the cluster over 2-channels, as shown in the bottom plot.

Another example is shown in figure 21(b), where a $150 \mu \mathrm{m}$ thick detector with $25 \mu \mathrm{m}$ strip pitch has been simulated. This is one of the options of the LHCb vertex detector. The vertex detector is used in the LHCb L1-trigger, for which a binary cluster search will be made with a $\mathrm{S} / \mathrm{N}$ threshold which can be as low as 3 with an expected noise per channel of 1000 ENC. Here the efficiency for the $p$-on- $n$ design would drop already for a $10 \%$ under-depletion, mainly due to the spreading of the cluster over many channels as a result of the small pitch compared to the thickness of the non-depleted layer. On top of that the resolution is expected to degrade quickly.

\section{Conclusions}

The performance of a heavily irradiated double-sided silicon microstrip detector operated at cryogenic temperatures has been characterised. It has been observed that for situations of incomplete depletion the $p$-side clusters broaden, leading to degradation of resolution and efficiency. A model has been developed which describes the observed behaviour, and allows predictions to be made for LHC detectors.

Wide variations in CCE have been observed in the irradiated area of the detector, depending on a number of operating conditions, which include bias voltage, temperature, time and bias voltage history. At temperatures below $130 \mathrm{~K}$ striking improvements in the CCE have been observed. By analysis of the cluster shapes it has been shown that these variations in $\mathrm{CCE}$ are related to variations in depletion depth, i.e. the voltage which is needed to fully deplete the detector changes as a function of the operating conditions. The CCE recovery is known as the "Lazarus effect" and has been discussed in other recent publications on diode detector data $[1,10]$. For the data described in this paper the "Lazarus effect" was observed and characterised but not controlled. In particular, the CCE improvements observed showed a decay in time. In principle it may be possible to control the "Lazarus effect" by manipulation of currents in the detector bulk. This approach is hinted at with the "partly forward biased" runs described in this paper, and addressed in the accompanying paper [2].

\section{Acknowledgements}

The work described in this paper resulted from a joint effort among people from the RD39 collaboration and the COMPASS, DELPHI and LHCb experiments. We thank the IPHELausanne, the LHEP-Bern and the CERN-EP Microelectronics workshops for technical support. We thank A. Hauska for participating in the setup preparation and S. Gerassimov for helpful discussions concerning the reference telescope. We are grateful to C. Buttar, P. Riedler and J. Wustenfeld for help with the ATLAS CERN-PS irradiation facility and for informative discussions. We thank L. Beattie, V. Eremin, Z. Li, E. Verbitskaya and S. Watts for illuminating discussions. K. Pretzl, K. Smith and P. Sonderegger are warmly acknowledged for their early and continuous support.

This research was supported by the Schweizer National-fonds zur Förderung der wissenshaftlichen Forschung (FORCE) and by the Stiftung zur Förderung der wis- 
a) $80 \mu \mathrm{m}$ strip pitch, binary

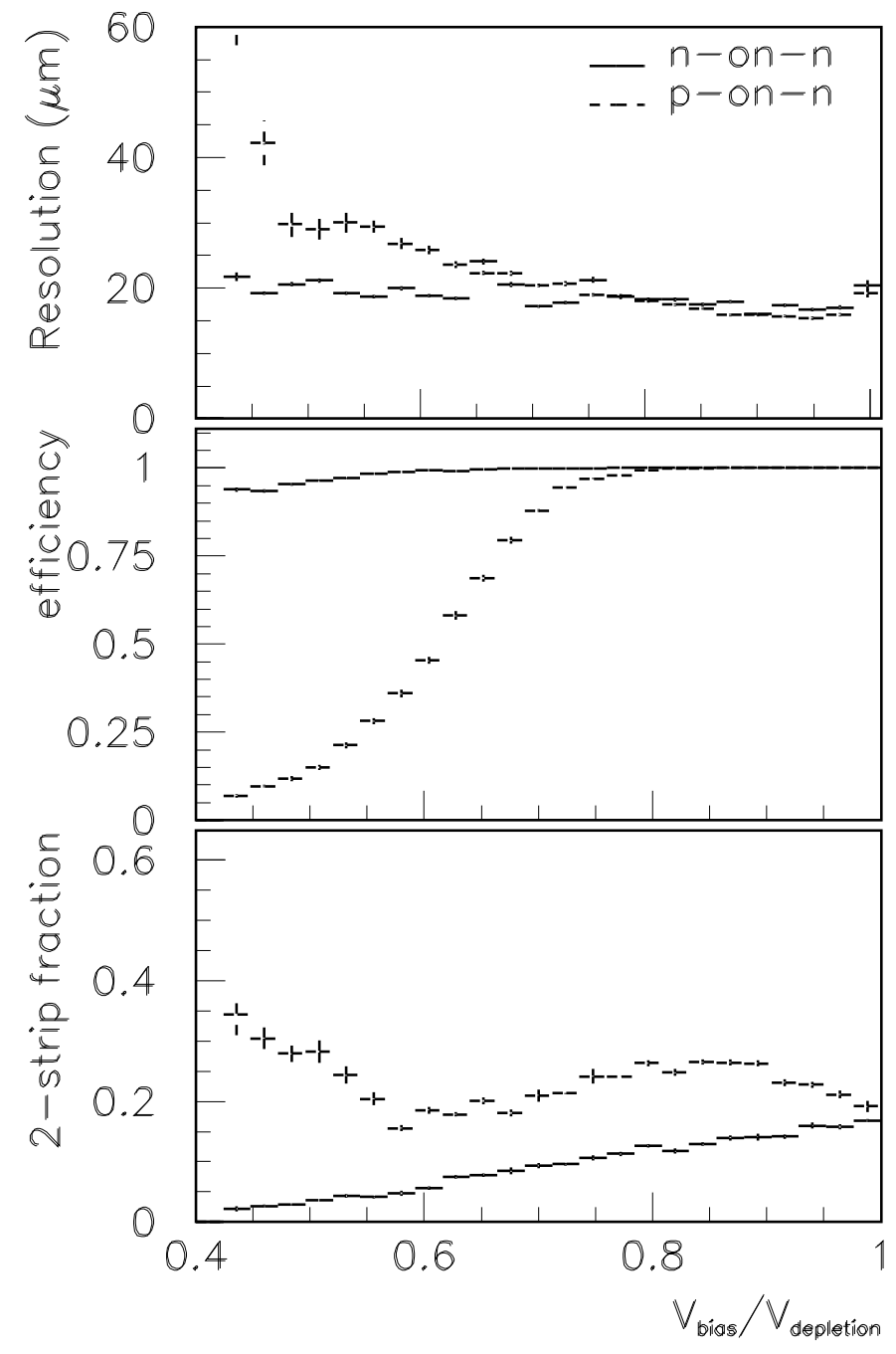

b) $25 \mu \mathrm{m}$ strip pitch, binary

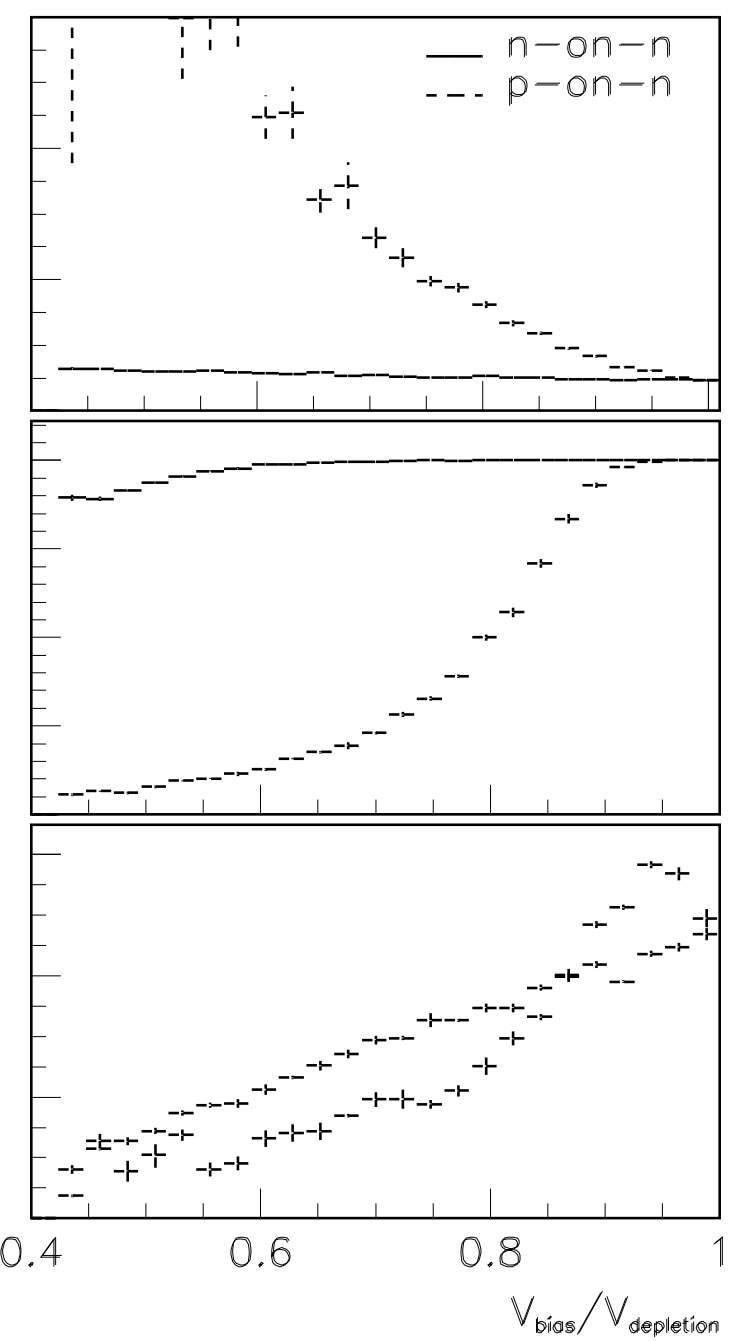

Figure 21: Expected performance for two different detector types with binary readout as a function of $V_{\text {bias }} / V_{\text {depletion. }}$ (a) shows a $300 \mu \mathrm{m}$ thick detector with $80 \mu \mathrm{m}$ strip pitch. A threshold of 6000 ENC for a noise of 1500 ENC per channel is assumed. (b) shows a $150 \mu \mathrm{m}$ thick detector, $25 \mu \mathrm{m}$ strip pitch and a threshold of $3000 \mathrm{ENC}$ for a noise per channel of 1000 ENC. 
senschaftlichen Forshung an der Universität Bern. One of the authors (L.C.) acknowledges financial support from the EC (TMR programme) under contract number ERBFMBICT961204.

\section{References}

[1] V.G. Palmieri et al., Evidence for charge collection efficiency recovery in heavily irradiated silicon detectors operated at cryogenic temperatures, Nucl. Instr. and Meth. A413 (1998) 475.

[2] K. Borer et al., Charge Collection Efficiency of Irradiated Silicon Detectors Operated at Cryogenic Temperatures, accompanying paper submitted to Nucl. Instr. and Meth., A.

[3] DELPHI Coll., P. Aarnio et al., Nucl. Instrum. Methods A 303, 233, (1991); DELPHI Coll., P. Abreu et. al., Nucl. Instrum. Methods A 37857 (1996).

[4] V. Chabaud et al., The DELPHI silicon strip microvertex detector with double-sided readout, Nucl. Instr. and Meth. A368 (1996) 314.

[5] P. Seller et. al., Noise Evaluation and Improvement of the LAL-RAL Microplex Readout Chip for the DELPHI Vertex Detector Nucl. Instr. and Meth. A315 (1992) 393.

[6] W. Bruckner et al., Silicon Microstrip Detectors with SVX Chip Readout, Nucl. Instr. and Meth. A348 (1994) 444.

[7] L. Brogiato et. al., Proceedings of the 3rd European Workshop on Low Temperature Electronics (WOLTE 3), Journal de Physique IV (Proceedings), Vol. 8, Pr 3, 1998

[8] L.W. Moyal, Theory of Ionization Fluctuations, Phil. Mag. 46 (1955) 263.

[9] L. Beattie et al., Charge collection efficiency in heavily irradiated silicon diodes , Nucl. Instr. and Meth. A412 (1998) 238-246.

[10] Z. Li et al., Improved Neutron Radiation Hardness for Si detectors: Application of Low Resistivity Starting Material and/or Manipulation of $N_{\text {eff }}$ by Selective Filling of Radiation-induced Traps at Low Temperatures, Presented at NSS 1998, Toronto, Canada, to be published in the proceedings.

[11] D. Morgan et al., Annealing of irradiated silicon strip detectors for the ATLAS experiment at CERN, Nucl. Instr. and Meth. A426 (1999) 336.

[12] A. Ruzin, Results from the ROSE collaboration Presented at VERTEX '99, Texel, The Netherlands, to be published in the proceedings.

[13] F. Lemeilleur et al., 2nd RD48 Status Report, LHCC 98-39, 1998.

[14] S.J. Watts, Radiation Induced Defects in Silicon, to be published in the proceedings of the 194th Meting of the Electrochemical Society Inc., Boston, MA, USA, November 1-6 1998 . 
[15] N. Bingefors et al., The DELPHI Microvertex detector, Nucl. Instr. and Meth. A328 (1993) 447.

[16] G. Cavalleri et al., Extension of Ramo's theorem as applied to induced charge in semiconductor detectors, Nucl. Instr. and Meth. 92 (1971) 137-140. 